\title{
Histone modifications affect differential regulation of TGF $\beta$ - induced NADPH oxidase 4 (NOX4) by wild-type and mutant p53
}

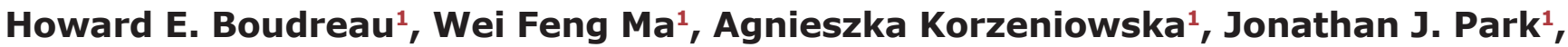 \\ Medha A. Bhagwat ${ }^{2}$ and Thomas L. Leto ${ }^{1}$ \\ ${ }^{1}$ Laboratory of Host Defenses, National Institute of Allergy and Infectious Diseases, National Institutes of Health, Bethesda, \\ Maryland, USA \\ ${ }^{2}$ Bioinformatics Support Program, National Institutes of Health Library, National Institutes of Health, Bethesda, Maryland, USA \\ Correspondence to: Thomas L. Leto, email: tleto@niaid.nih.gov \\ Keywords: mutant p53, NOX4, p300, histone modification, cell migration \\ Received: December 16, $2016 \quad$ Accepted: April 26, $2017 \quad$ Published: May 16, 2017
}

Copyright: Boudreau et al. This is an open-access article distributed under the terms of the Creative Commons Attribution License 3.0 (CC BY 3.0), which permits unrestricted use, distribution, and reproduction in any medium, provided the original author and source are credited.

\section{ABSTRACT}

Previously, we showed wild-type (WT) and mutant (mut) p53 differentially regulate reactive oxygen species (ROS) generation by NADPH oxidase-4 (NOX4): p53WT suppresses TGF $\beta$-induced NOX4, ROS and cell migration, whereas tumor-associated mut-p53 proteins enhance NOX4 expression and cell migration. Here, we extended our findings on the effects of p53 on NOX4 in several tumors and examined the basis of NOX4 transcriptional regulation by $\mathrm{p} 53$ and SMAD3. Statistical analysis of expression data from primary tumors available from The Cancer Genome Atlas (TCGA) detected correlations between mut-p53 and increased NOX4 expression. Furthermore, by altering p53 levels in cell culture models we showed several common tumor-associated mutant forms support TGF $\beta$ /SMAD3-dependent NOX4 expression. Deletion analysis revealed two critical SMAD3 binding elements (SBE) required for mut-p53-dependent NOX4 induction, whereas p53-WT caused dose-dependent suppression of NOX4 transcription. ChIP analysis revealed SMAD3 and p53-WT or mut-p53 associate with SBEs and p53 response elements in a TGF $\beta$-dependent manner. Interestingly, the repressive effects of p53-WT on NOX4 were relieved by mutation of its transactivation domain or histone deacetylase (HDAC) inhibitor treatment. Overexpression of p300, a transcriptional co-regulator and histone acetyltransferase (HAT), enhanced p53-mediated NOX4 induction, whereas HAT-inactive p300 reduced NOX4 expression. Mut-p53 augmented TGF $\beta$-stimulated histone acetylation within the NOX4 promoter. Finally, wound assays demonstrated NOX4 and p300 promote TGF $\beta /$ mut-p53-mediated cell migration. Our studies provide new insight into TGF $\beta$ /SMAD3 and mut-p53-mediated NOX4 induction involving epigenetic control of NOX4 in tumor cell migration, suggesting NOX4 is a potential therapeutic target to combat tumor progression and metastasis.

\section{INTRODUCTION}

The tumor suppressor gene TP53 is the most commonly mutated gene in human cancers. Approximately $50 \%$ of all human cancers produce an inactive mutated tumor suppressor protein [1]. Tumorassociated p53 mutations are primarily missense mutations within the DNA binding domain that can give rise to a dominant-negative protein or a protein that has taken on a gain-of-function (GOF) with pro-oncogenic effects [1, 2]. Tumor-associated p53 mutants have been shown to support an increase in cell proliferation, invasion, migration, angiogenesis, resistance to chemotherapeutic drugs, and tumor development in animal models [3-7]. Several reports have demonstrated that expression of GOF p53 mutants in p53-null cell models results in up-regulation of genes associated with cell survival, proliferation, and migration, whereas genes involved in cell cycle arrest and apoptosis are downregulated [8, 9]. However, the molecular mechanisms 
underlying gene expression changes that support the GOF phenotypes are still unclear.

Transforming growth factor-beta (TGF $\beta$ ) is a pluripotent cytokine that can have either tumorsuppressing or tumor-promoting effects. As tumors progress, they respond to TGF $\beta$ by increasing their mobility and invasiveness, developing a more metastatic phenotype. Studies have shown that p53 plays an important role in TGF $\beta / \mathrm{SMAD} 2 / 3$-mediated cell signaling and migration [10-12]. The switch between TGF $\beta$ being a tumor suppressor to a tumor promoter was shown to involve mut-p53 and SMAD2 forming a complex with p63 that inhibits p63 tumor suppressor function $[11,13]$. Another recent study demonstrated an ERK-mediated interaction between mut-p53 (R175H) and SMAD3, but not SMAD2, regulates subsets of TGF $\beta$ target genes that promote cell migration and invasion [13]. Conversely, Cordenonsi et al. demonstrated p53-WT/SMAD2/3 complexes promote cell cycle arrest and apoptosis [14].

While studies have shown mut-p53 recognizes different regulatory sequences of its target genes, it is still unclear whether mut-p53 has a distinct DNA-binding consensus sequence. Several groups have reported on proteins that interact with mutant GOF p53, and found many of them are transcription factors and co-regulators, suggesting mut-p53 may indirectly regulate transcription through recruitment and complex formation [15]. Recent studies have provided evidence that GOF mut-p53 proteins bind to and increase the expression of chromatin regulatory genes such as methyltransferases and acetyltransferases, thereby increasing histone methylation and acetylation, and subsequently favoring a pro-oncogenic transcriptional program and phenotype $[16,17]$.

The transcriptional co-activator p300 is a histone acetyltransferase (HAT) that can acetylate histones and several transcription factors. A recent report suggested mut-p53 enhanced gene expression through recruitment and interaction with p300 and SMAD3 in many cancer cell types [17]. Contrary to this, they found that p53-WT associates with histone deacetylases (HDAC) in a corepressor complex inhibiting gene expression [18, 19]. Another study showed phosphorylated p53-R175H binds to $\mathrm{p} 300$, strengthening transcriptional activity [20].

Previously, we showed wild-type and mutant forms of p53 differentially regulate reactive oxygen species (ROS) generation by NADPH oxidase 4 (NOX4) in breast and lung epithelial tumor lines [21]. We found that p53-WT suppresses TGF $\beta$-induced NOX4, ROS production, and cell migration, whereas tumor-associated mut-p53 proteins (R175H and R280K) enhance NOX4 expression and cell

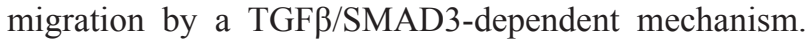
The mechanisms underlying TGF $\beta /$ SMAD3/p53-based NOX4 regulation remain elusive. However, a recent report shed light on TGF $\beta$-induced NOX4 gene expression by identifying a critical SMAD3 binding site in an upstream NOX4 transcriptional regulatory sequence [22].
Furthermore, histone modifications by acetlylation and methylation were recently shown to play an important role in epigenetic regulation of NOX4 gene expression [23].

Here, we examined the basis of NOX4 promoter regulation by $\mathrm{p} 53$ and SMAD3. Analysis of upstream NOX4 transcriptional regulatory sequences, identified critical SMAD3 binding elements (SBE) and p53 response elements (p53RE) required for mut-p53-induced NOX4 expression. Moreover, expression of active SMAD3 results in robust NOX4 promoter activity, which is abolished when co-expressed with $\mathrm{p} 53-\mathrm{WT}$. The repressive effect by p53-WT on NOX4 is relieved upon treatment with HDAC inhibitors. Furthermore, overexpression of p300, a known mut-p53-binding transcriptional co-regulator with HAT activity, enhances mut-p53-mediated NOX4 promoter activity and cell migration. Together these results provide further insight on regulation and epigenetic control of NOX4 by TGF $\beta /$ SMAD3 and p53.

\section{RESULTS}

\section{Differential regulation of TGF $\beta$-induced NOX4 by wild-type and mutant p53}

To elucidate the mechanisms involved in p53 regulation of NOX4, we first sought to confirm whether p53 mutation status is correlated with NOX4 mRNA expression in different primary tumor samples. We performed statistical analysis on gene expression data available from TCGA. Here we found a correlation between commonly occurring p53 missense mutations within the "hot-spot" DNA binding domain region and increased expression of NOX4 in several tumor types. Breast invasive carcinoma samples with p53 mutations exhibited higher NOX4 expression relative to tissues with p53-WT (Figure 1A). However, tumor samples with mut-p53-Y220C correlated with reduced NOX4 expression indicating that not all DNA binding domain p53 mutants result in higher NOX4 expression. Further, p53 mutations in pancreatic adenocarcinoma and head and neck squamous cell carcinoma also were correlated with increased NOX4 expression relative to their WT counterparts (Figure 1A).

TGF $\beta$ is a well-known inducer of the epithelial-tomesenchymal transition (EMT) transcriptional program involving SMAD3 activation, whereby genes involved in cell-cell junctions and epithelial polarization are downregulated while genes associated with the extracellular matrix and migration such as fibronectin are upregulated [24]. Previously, we examined TGF $\beta$-induced fibronectin expression in relation to NOX4 and found p53WT suppressed TGF $\beta$ induction of fibronectin, similar to NOX4 [21]. Here, we sought further evidence supporting the correlation between NOX4 and fibronectin expression patterns in relation to p53 mutation status in tumor samples from TCGA. Interestingly, increased NOX4 and fibronectin mRNA expression positively correlated in 
Breast Invasive Carcinoma

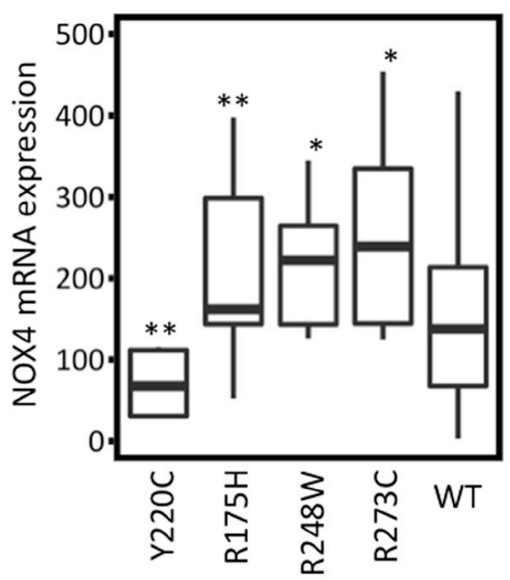

B
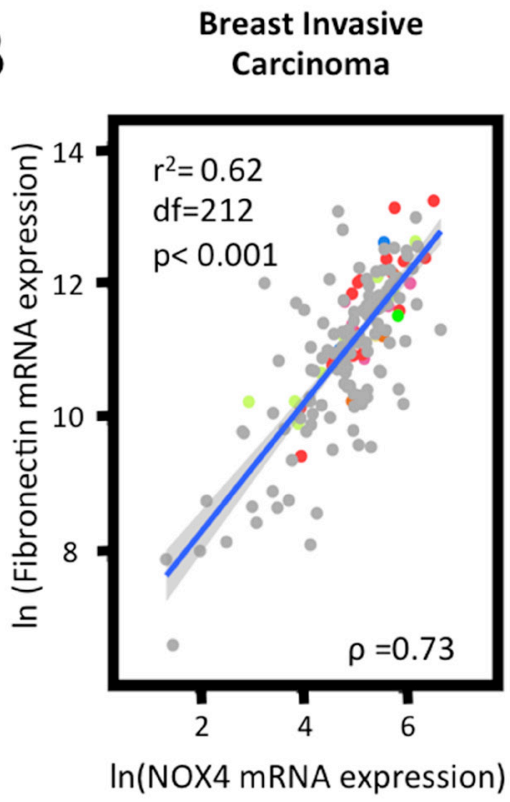

Pancreatic Adenocarcinoma

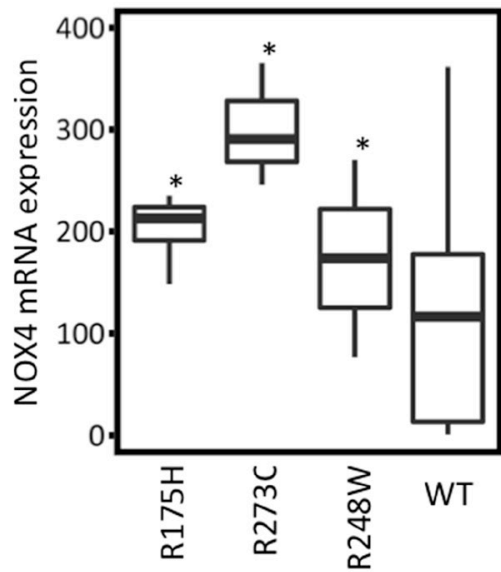

Pancreatic Adenocarcinoma

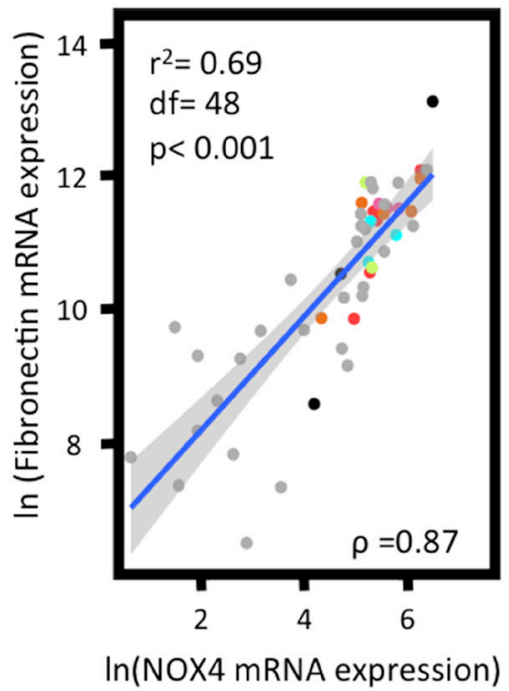

Head and Neck Squamous Cell Carcinoma

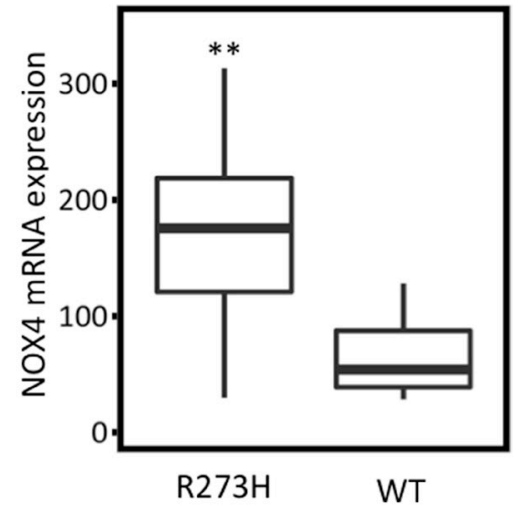

Head and Neck Squamous Cell Carcinoma

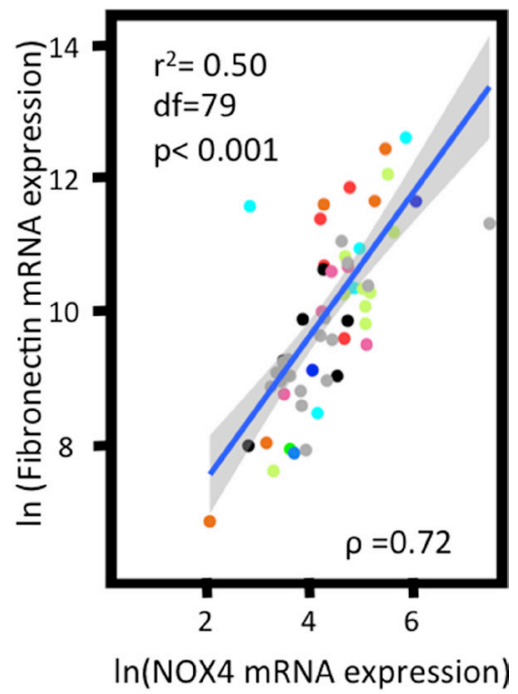

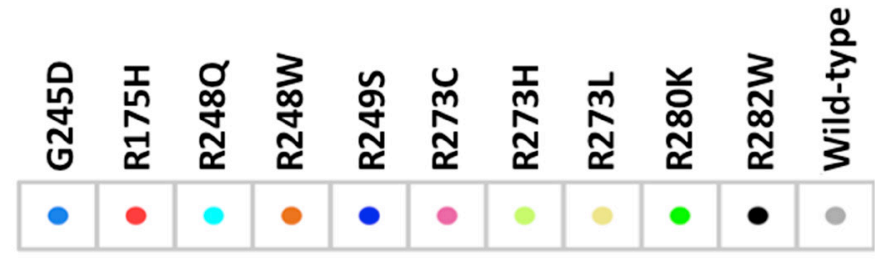

p53 missense mutations

Figure 1: TP53 mutation status correlates with NOX4 expression. Clinical sample data from TCGA were extracted via cBioPortal. Samples were grouped by cancer studies and by p53 mutation status. (A) Breast invasive carcinoma (R175H [ $n=42], \mathrm{R} 248 \mathrm{~W}[n=14]$, R273C [ $n=12]$, Y220C [ $n=11]$, wild-type [ $n=116]$ ), pancreatic adenocarcinoma (R175H [ $n=5]$, R273C [ $n=3]$, R248W [ $n=5]$, wildtype $[n=29])$ and head and neck squamous cell carcinoma (R273H $[n=16]$ and wild-type $[n=17])$ NOX4 mRNA expression in samples with different p53 mutations were compared to those with p53-WT within their respective cancer studies. Statistical significance was established using Mann-Whitney test where appropriate. Extreme outliers were removed from plots but retained during statistical analysis. (B) Increases in NOX4 mRNA expression are positively correlated with fibronectin expression patterns. TCGA data were analyzed using Spearman rank correlation tests and linear regression analysis. Data points were colored based on the assigned p53 mutation status. The R script, copies of the data used and high-resolution figures are publically available at github.com/wfma/HEBoudreau. Significance values are indicated as $* P$-value $<0.05$, or $* * P$-value $<0.01$. 
tissue samples with p53 DNA binding domain 'hot-spot" mutations (Figure 1B). Collectively, these data suggest mut-p53-induced NOX4 has a role in cancer progression in several tumor types.

We then explored the divergent effects of wild-type and mut-p53 on NOX4 expression in several established tumor cell models. We transfected p53-null Hep3B hepatocytes with p53-WT followed by TGF $\beta$ stimulation for 24 hours. Expression of p53-WT significantly reduced TGF $\beta$-induced $\mathrm{NOX} 4$ at both the mRNA and protein levels (Figure 2A). Next, we transfected PLC/ $\mathrm{PRF} / 5$ hepatocytes with $\mathrm{p} 53$-specific siRNAs to deplete these cells of the endogenously expressed p53-R249S. Knockdown of p53-R249S significantly reduced both TGF $\beta$-induced NOX4 mRNA and protein expression (Figure 2B). We further confirmed our results in HepG2 cells, which express endogenous p53-WT. Depletion of p53-WT in HepG2 cells enhanced NOX4 expression in the presence or absence of TGF $\beta$ (Figure 2C). We also observed a decrease in TGF $\beta$-induced NOX4 expression in PANC-1 pancreatic cancer cells upon silencing of endogenous p53-R273H (Figure 2D). Together, these results confirm that $\mathrm{p} 53$-WT suppresses whereas mut-p53 enhances TGF $\beta$-mediated NOX4 expression in both hepatic and pancreatic tumor models.

\section{Several tumor-associated p53 mutants increase NOX4 mRNA and promoter activity in a TGF $\beta$ - dependent manner}

We have previously shown that $\mathrm{p} 53-\mathrm{R} 175 \mathrm{H}$ and p53-R280K enhanced TGF $\beta$-induced NOX4 expression; here we examined other common tumor-associated p53 mutants on NOX4 gene expression (Figure 3A). First, we investigated NOX4 mRNA expression in response to TGF $\beta$ in Hep3B (p53-null) cells heterologously expressing p53WT, p53-R175H, p53-R248Q, p53-R273H, p53-D281G or control plasmids (Figure 3B). Control cells and p53 mutant expressing cells treated with TGF $\beta$ for 24 hours displayed robust increases in NOX4 mRNA whereas expression of p53-WT diminished this effect. Interestingly, p53-R175H and p53-D281G mutants increased NOX4 mRNA in the absence of TGF $\beta$ stimulation. Furthermore, p53-D281G augmented TGF $\beta$-induced NOX4 expression compared to treated control cells. These effects were confirmed in H1299 (p53-null) lung epithelial cells. H1299 cells expressing p53WT, p53-R175H, p53-R248Q, p53-R249S, p53-R273H, p53-R280K, p53-D281G or control vectors were treated with TGF $\beta$ for 24 hours. NOX4 mRNA expression was substantially up-regulated in cells expressing mut-p53 compared to $\mathrm{p} 53-\mathrm{WT}$ with or without TGF $\beta$ stimulation (Figure 3C). Similar to the Hep3B hepatocytes, p53-D281G caused the greatest enhancement of NOX4 expression upon TGF $\beta$ treatment compared to control.

Next, we examined the effect of p53-WT and mutant expression on the NOX4 promoter. A sequence of 4,760 base pairs upstream from the NOX4 transcriptional start site cloned into a luciferase reporter plasmid (pGL3) [22] was used to measure promoter activity. Hep3B cells co-transfected with the NOX4 promoter-reporter and increasing concentrations of mut-p53 plasmids (p53$\mathrm{R} 175 \mathrm{H}, \mathrm{p} 53-\mathrm{D} 281 \mathrm{G}$ ) displayed a dose-dependent increase in NOX4 promoter activity in comparison to pGL3-Basic empty control transfected cells (Figure 3D). These results show mut-p53 proteins increased NOX4 promoter activity even in the absence of TGF $\beta$. However, we found mut-p53mediated NOX4 promoter activity was TGF $\beta$-dependent, since NOX4 promoter (-4760) activity induced by p53R175H or p53-D281G was abolished with a TGF $\beta$ R1specific inhibitor, 616451, or a SMAD3 specific inhibitor, SIS3 (Figure 3E). To further validate the role of TGF $\beta$ / SMAD3 signaling on NOX4 promoter activation, we cotransfected the NOX4 promoter (-4760) with constitutively active forms of SMAD2 or SMAD3. A significant increase in promoter activity was observed in cells expressing constitutively active SMAD3, whereas active SMAD2 had minimal effect on the NOX4 promoter (Figure 3F). Collectively, these data demonstrate that several of the common tumor-associated mut-p53 proteins positively regulate NOX4 at both the mRNA and promoter level in a TGF $\beta /$ SMAD3-dependent manner, whereas p53-WT acts as a potent repressor of TGF $\beta$-mediated NOX4 expression.

\section{Wild-type p53 is a potent repressor of TGFß/ SMAD3-induced NOX4 promoter activity}

We further explored the repressive effect p53-WT has on NOX4 promoter function and found p53-WT diminished TGF $\beta$ activation of the NOX4 promoter in a dose-dependent manner (Figure 4A). Similarly, when cotransfected with constitutively active SMAD3, NOX4 promoter activity $(-4760)$ was diminished by increasing p53-WT expression (Figure 4B). Moreover, NOX4 promoter activation in cells expressing constitutively active TGF $\beta$ R1 (T204D) was significantly blunted when co-expressed with p53-WT in Hep3B (Figure 4C) and in H1299 (Figure 4D) p53-null cell models.

Next, we compared the expression of NOX4 to p21/ CDKN1A, a well-established activated p53 target gene involved in cell cycle arrest. We treated HepG2 cells which express endogenous p53-WT with 5-Fluorouracil (5-FU), a chemotherapeutic drug that induces p53dependent cell growth arrest and apoptosis [25]. We found that $\mathrm{p} 21$ gene expression was induced in a 5 -FU dose-dependent manner, but not in cells treated with TGF $\beta$ (Figure 4E, left). Conversely, NOX4 expression was induced by TGF $\beta$ but not by 5 -FU treatments (Figure 4E, right). Moreover, in Hep3B cells co-transfected with p53-WT and a p21 promoter reporter plasmid, p21 promoter activity was significantly increased with or without 5-FU, whereas p53-WT and 5-FU has no effect on the NOX4 promoter (Figure 4F). Taken together, these 

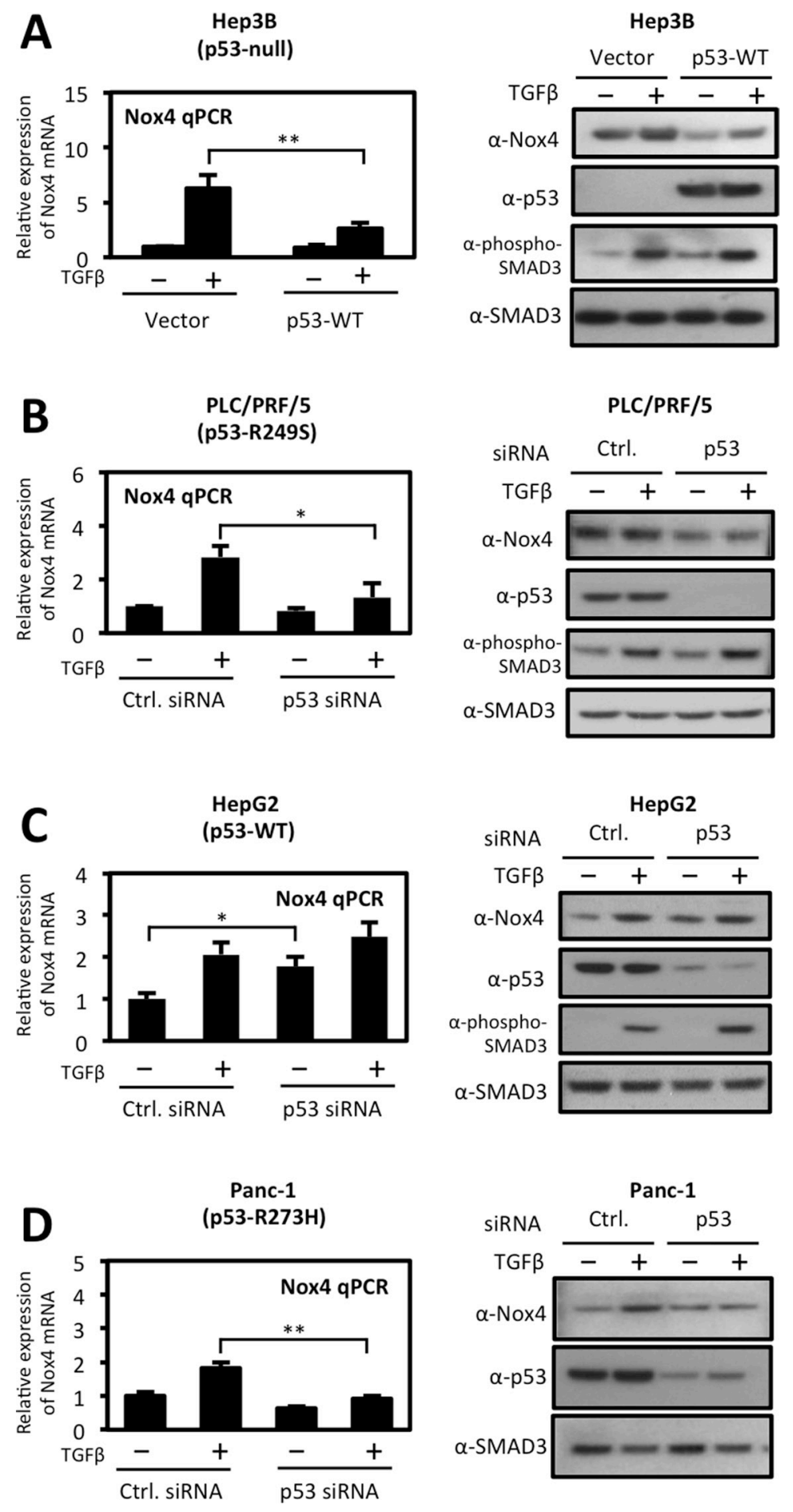

Figure 2: Differential regulation of TGFß-induced NOX4 by wild-type and mutant p53. (A) Left panel, p53-null Hep3B cells were transfected with vector control or $\mathrm{p} 53$-WT plasmids. Twenty-four hours after transfection, cells were treated with TGF $\beta$ ( $5 \mathrm{ng} / \mathrm{ml})$ for 24 hours. Real-time quantitative PCR (qPCR) analysis of mRNA expression was determined using human NOX4-specific primers. GAPDH-specific primers were used for a reference gene for normalization. Quantification of NOX4 mRNA is described relative to vector untreated cells. Right panel, Hep3B cells were transfected and treated as the left panel. Thirty micrograms of total cell lysates were analyzed by western blotting. The immunoblot was probed sequentially with antibodies against NOX4, p53, phospho-SMAD3, and total SMAD3. (B, C and D) Effects of siRNA-mediated silencing of endogenous wild-type or mut-p53 on NOX4 expression. (B) PLC/PRF/5 (p53-R249S), (C)

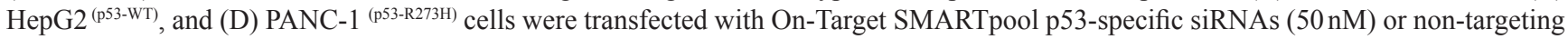
control siRNAs $(50 \mathrm{nM})$ or for $48 \mathrm{~h}$ followed by TGF $\beta(5 \mathrm{ng} / \mathrm{ml})$ treatment for an additional $24 \mathrm{~h}$. Quantitative PCR of NOX4 mRNA (left panel) and protein expression (right panel) were analyzed as in panel A $(n=3)$. Significance values are indicated as $* P$-value $<0.05$, or $* * P$-value $<0.01$. 
A

Tumor-associated p53 mutations

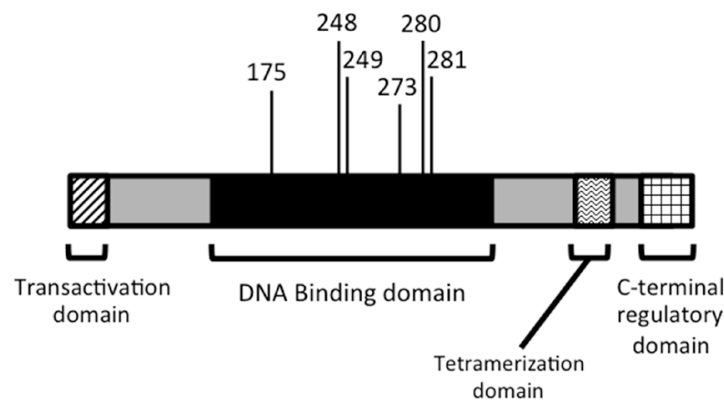

B
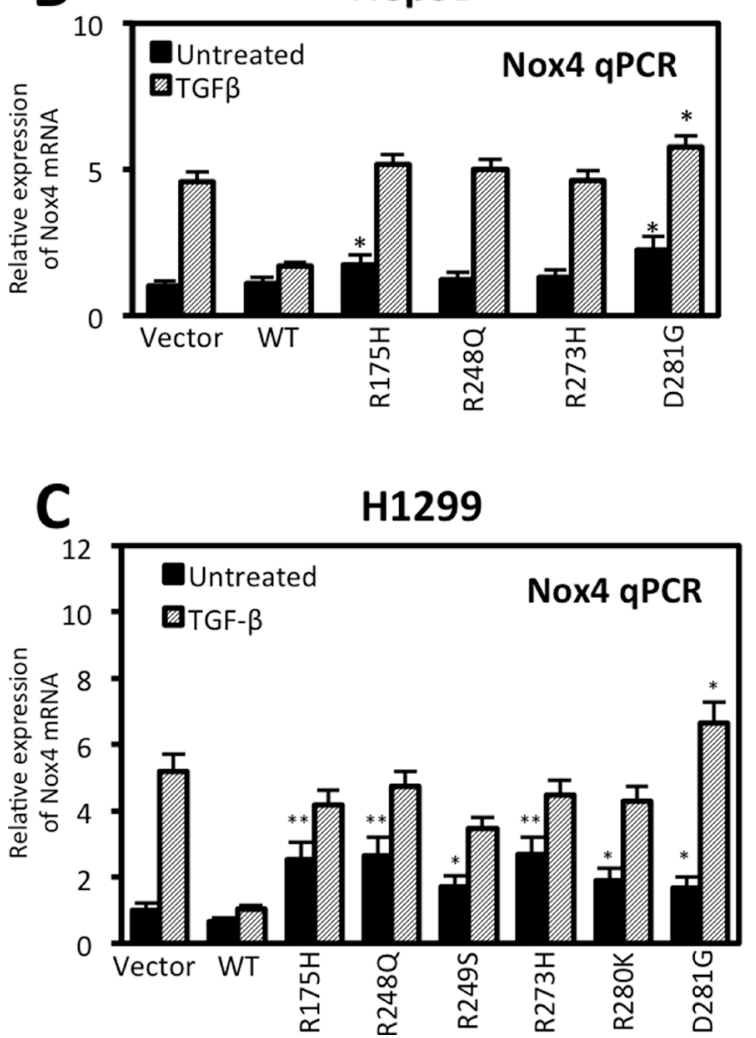

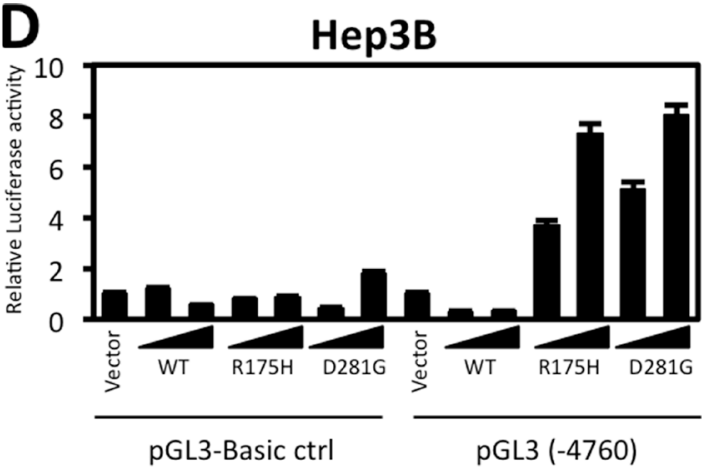

E
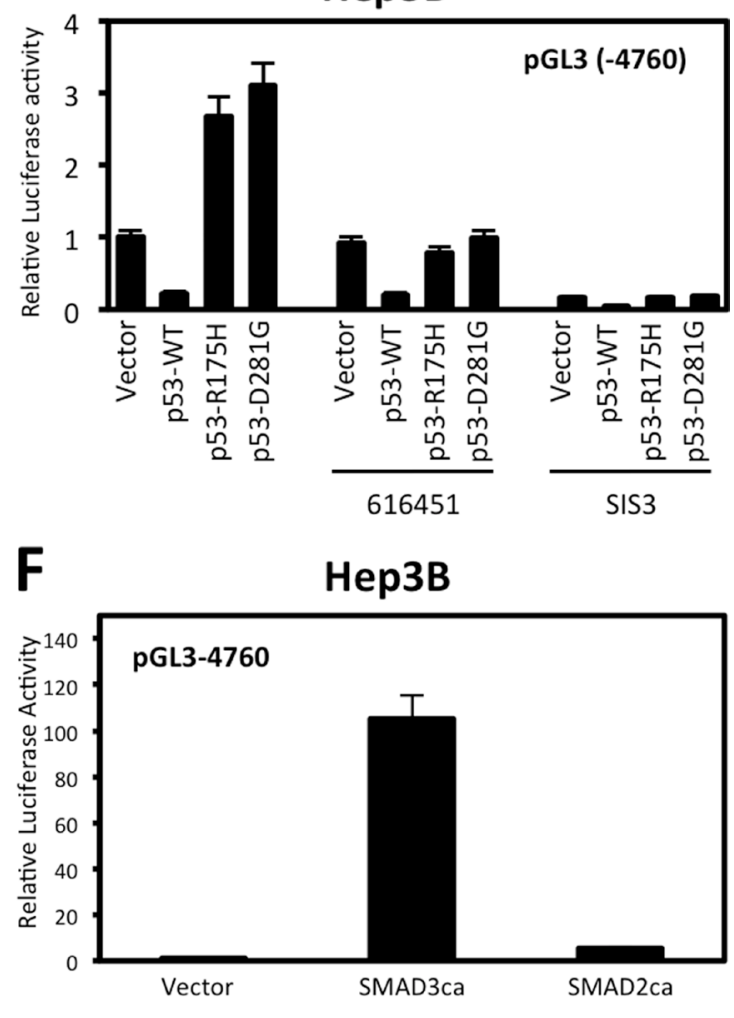

Figure 3: Tumor-associated p53 mutants increase NOX4 mRNA and promoter activity in a TGF (A) Schematic representation of the p53 functional domains showing common tumor-associated "hotspot" missense mutations within the DNA binding domain. (B) Hep3B cells were transfected with control, p53-WT, p53-R175H, p53-R248Q, p53-R273H or p53-D281G plasmids. Twenty-four hours after transfection, the cells were treated with TGF $\beta(5 \mathrm{ng} / \mathrm{ml})$ or left untreated for an additional 24 hours. NOX4 mRNA expression was determined by qPCR analysis using NOX4 and GAPDH-specific primers. Results for NOX4 mRNA are quantified relative to vector untreated control $(n=3$, in triplicate). (C) H1299 cells were transfected with vector control plasmid, p53-WT, p53R175H, p53-R248Q, p53-R249S, p53-R273H, p53-R280K, or p53-D281G mutant plasmids. The cells were then treated and analyzed for NOX4 mRNA expression as in panel B ( $n=3$, in triplicate). (D) Hep3B cells were co-transfected with NOX4 promoter luciferase reporter plasmid pGL3-NOX4 (-4760) or pGL3-Basic control $(0.5 \mu \mathrm{g})$ and increasing amounts $(0.25 \mathrm{or} 0.5 \mu \mathrm{g})$ of vector plasmids or p53-WT, p53- R175H, or p53-D281G plasmids for 48 hours. Total cell lysates were collected and assayed for luciferase activity by luminescence. Luciferase activity is described as fold change in relative light units compared to the vector untreated cells $(n=3$, in triplicate). (E) Hep3B cells were co-transfected with NOX4 promoter luciferase reporter plasmid pGL3-NOX4 (-4760) $(0.5 \mu \mathrm{g})$ and vector control plasmids or p53-WT, p53- R175H, or p53-D281G plasmids. After 24hours, the cells were treated with $616451(10 \mu \mathrm{M})$, a TGF $3 \mathrm{R} 1$-specific inhibitor or SIS3 $(10 \mu \mathrm{M})$, a SMAD3-specific inhibitor for $4 \mathrm{~h}$ before treating with TGF $\beta$ for 20 hours. Cell lysates were collected and assayed for luciferase activity by luminescence ( $n=3$, in triplicate). (F) Hep3B cells were co-transfected with NOX4 promoter luciferase reporter plasmid and control plasmid, SMAD3 constitutively active (SMAD3ca), or SMAD2 constitutively active (SMAD2ca) plasmids. Fortyeight hours post-transfection, cell lysates where analyzed for luciferase activity $(n=3$, in triplicate). Significance values are indicated as $* P$-value $<0.05$, or $* * P$-value $<0.01$. 
A
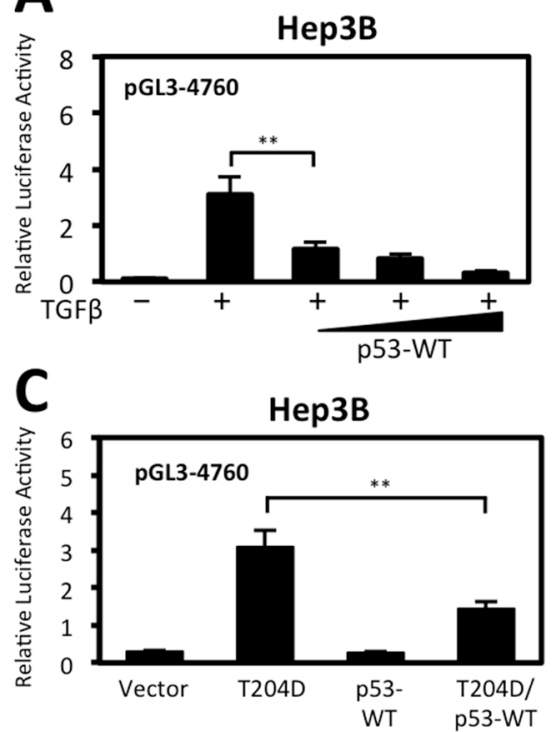

E

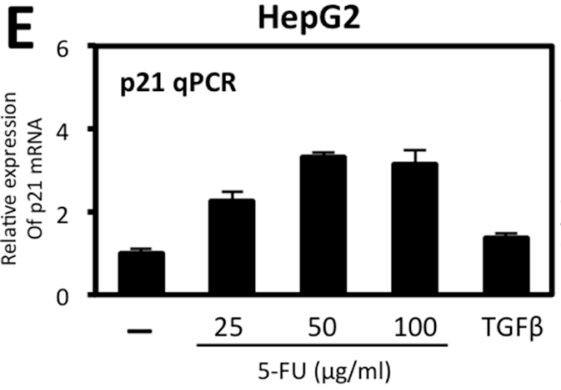

B
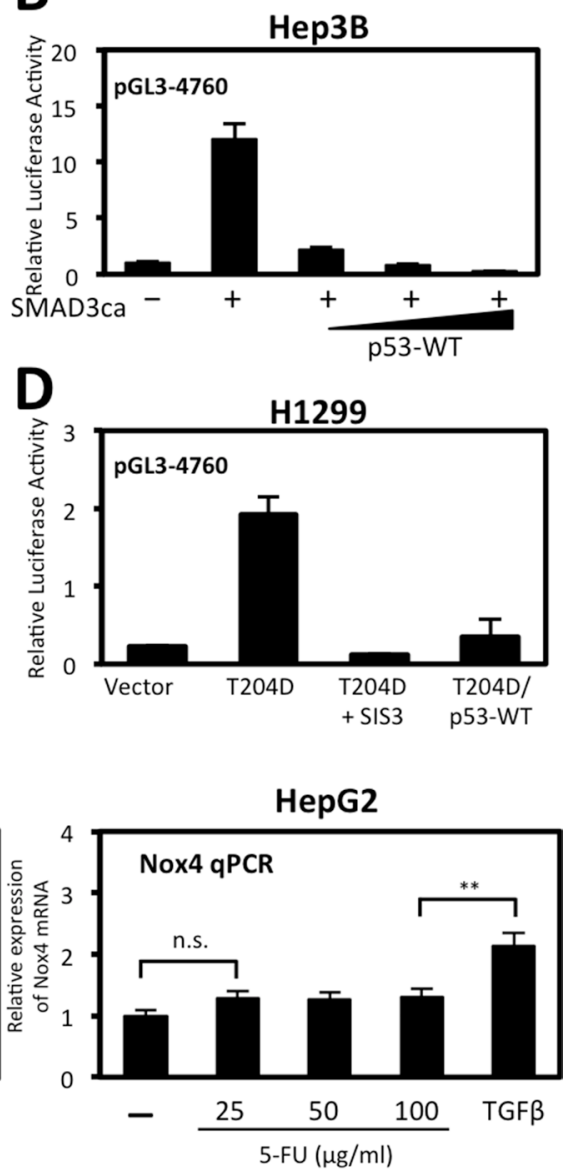

F

Hep3B

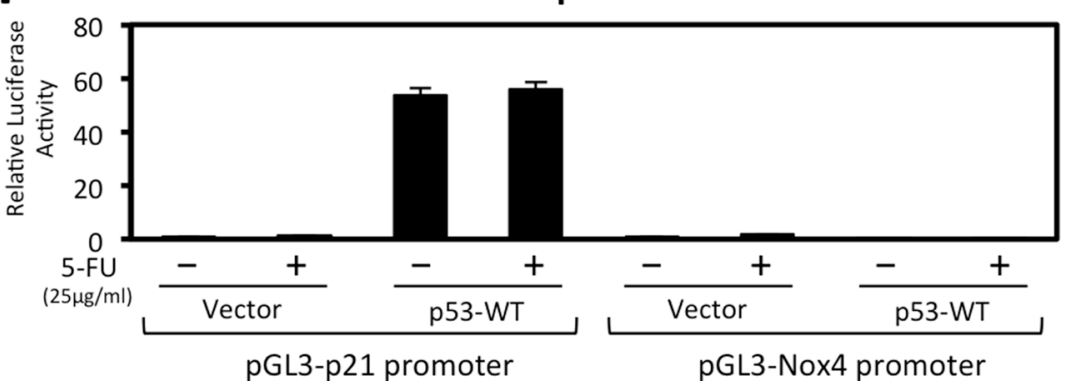

Figure 4: Wild-type p53 represses TGFB/SMAD3-induced NOX4 promoter activity. (A) Hep3B cells were co-transfected with NOX4 promoter-luciferase reporter plasmid pGL3-NOX4 (-4760) $(0.5 \mu \mathrm{g})$ and vector control $(0.5 \mu \mathrm{g})$ or increasing amounts of p53-WT $(0.1,0.3$, or $0.5 \mu \mathrm{g})$ for 24 hours then either treated with TGF $\beta(5 \mathrm{ng} / \mathrm{ml})$ for 24 hours or left untreated. Total cell lysates were collected 48 hours post-transfection and assayed for luciferase activity by luminescence $(n=4$, in triplicate). (B) Hep3B cells were co-transfected with NOX4 promoter reporter and vector control or constitutively active SMAD3 (0.5 $\mu \mathrm{g})$. Wild-type p53 was co-transfected at increasing concentrations as in panel A. Forty-eight hours after transfection, total cell lysates were assayed as in panel A ( $n=3$, in triplicate). (C) Hep3B cells were co-transfected with pGL3-NOX4 (-4760) and either vector control, constitutively active TGFßR1 (T204D), p53-WT, or T204D and p53-WT. After 48 hours, total cell lysates were collected for luciferase activity ( $n=3$, in triplicate). (D) H1299 cells transfected with the pGL3-NOX4 (-4760) promoter-reporter were co-transfected with either vector control, T204D, or with T204D and p53-WT. Twenty-four hours after transfection, SIS3 $(10 \mu \mathrm{M})$ was added for an additional 24 hours. Cell lysates were collected and assayed for luciferase activity. (E) HepG2 cells were treated with increasing concentrations of 5-fluorouracile (5-FU) (25, 50, or 100 $\mu \mathrm{g} / \mathrm{ml})$ or TGF $\beta$ $(5 \mathrm{ng} / \mathrm{ml})$ for 24 hours. Real-time quantitative PCR (qPCR) analysis of p21/CDKN1A mRNA expression (right panel) or NOX4 mRNA (left panel) was determined using gene-specific primers for human p21 or NOX4. Results are described as p21 or NOX4 mRNA expression relative to untreated control and normalized to GAPDH $(n=3$, in triplicate). (F) Hep3B cells were co-transfected with p21 promoter reporter or pGL3-NOX4 (-4760) plasmids and control vector or p53-WT plasmids for 24 hours followed by 5 -FU (25 $\mu \mathrm{g} / \mathrm{ml})$ treatment for an additional 24 hours. Total cell lysates were collected and assayed for luciferase activity ( $n=3$, in triplicate). Significance values are indicated as $* * P$-value $<0.01$ or n.s. (not significant). 
data demonstrate $\mathrm{p} 53$-WT is a negative regulator of TGF $\beta$ induced NOX4 promoter activity and that NOX4 is not a target gene responsive to $\mathrm{p} 53-\mathrm{WT}$ activation by 5 -FU.

\section{The NOX4 promoter contains unique TGF $\beta$ / SMAD3 and p53 regulatory sequences}

Previous studies have indicated p53 and SMAD3 proteins can form a complex that regulates TGF $\beta$ responsive genes [11]. Moreover, recent studies by Bai et al. identified a TGF $\beta / \mathrm{SMAD}$-responsive region of the NOX4 gene located between -3976 bp and $-4760 \mathrm{bp}$ upstream of the transcription start site and an AP-1/SMAD binding site located at $-4667 \mathrm{bp}$ to $-4653 \mathrm{bp}$ critical for TGF $\beta$-mediated NOX4 promoter activity [22]. These findings prompted us to search for $\mathrm{p} 53$ response elements (RE) in close proximity to consensus SMAD binding elements (SBE). Several reports have shown that both wild-type and mutant p53 can mediate gene transcription using half-site response elements [26, 27]. We identified sequences that conform to consensus sequence for three half-site p53-REs and an additional SBE within the -3976 bp and -4760 bp promoter region of NOX4 (Figure 5A).

We analyzed a series of NOX4 deletion constructs to determine p53 and SMAD3 responsive regions (Figure 5A). Co-transfection of constitutively active SMAD3 and pGL3-NOX4 (-4760) resulted in robust promoter activity that was decreased in cells expressing the truncated pGL3-NOX4 (-3975) reporter construct (Figure 5B, 5C). SMAD3-induced promoter activity was completely diminished when co-transfected with p53-WT. Moreover, mut-p53 (p53-R175H and p53D281G) expression supported TGF $\beta$-stimulated (-4760) promoter activity. However, this activity was lost with cotransfection of the (-3975) reporter construct (Figure 5D). These data indicate p53-WT is a potent negative regulator of TGF $\beta / S M A D 3$-mediated transcription of NOX4, whereas mut-p53 supports NOX4 expression predominately through a TGF $\beta$-dependent mechanism.

\section{Deletion of conserved SMAD binding elements or p53 response elements reduce NOX4 promoter activity induced by TGF $\beta$}

To explore contributions of putative SMAD binding and p53 response elements, we generated pGL3-NOX4 $(-4760)$ promoter reporter constructs with deletions of either p53-1/SBE-1 overlapping sequences, p53-RE-2, p53-RE-3, or SBE-2 sequences (Figure 6A). Deletion of either the overlapping p53-1/SBE-1 or SBE-2 sequences completely abolished NOX4 promoter activity, whereas deleting p53-RE-2 or p53-RE-3 resulted in minimal changes in promoter activity (Figure 6B). Therefore, the overlapping SBE-1/p53-RE-1 and SBE-2 sequences are critical for TGF $\beta$-mediated promoter activity.
SMAD3 and p53 associate with NOX4 SBE and p53-RE sequences in a TGF $\beta$-dependent manner

Next, we address molecular mechanisms involved in p53 and SMAD3 regulation of NOX4. We used p53 and SMAD3 specific antibodies to perform chromatin immunoprecipitation (ChIP) assays in Hep3B cells transfected with p53-WT, p53-D281G, or control vector followed by 24 hours TGF $\beta$ stimulation. We found that p53-WT and SMAD3 were recruited to NOX4 sequences in the absence of TGF $\beta$, whereas p53-D281G and SMAD3 were recruited only in the presence of TGF $\beta$ (Figure 7A-7C). Further, SBE-1/p53-1 and SBE-2/p53-3 response elements were required for $\mathrm{p} 53-\mathrm{WT}$ and SMAD3 recruitment in the absence of TGF $\beta$, whereas SBE-2/p533 elements were necessary for $\mathrm{p} 53-\mathrm{D} 281 \mathrm{G}$ and SMAD3 recruitment in response to TGF $\beta$.

Next, we examined whether the p53 transactivation domain of either wild-type or mutant was critical in regulating NOX4. Mutation of hydrophobic amino acids (L22Q/W23S) within the transactivation domain of p53 abolish co-factor binding and transactivation of target genes $[28,29]$. Surprisingly, expression of the p53-L22Q/ W23S double mutant was unable to repress TGF $\beta$-mediated NOX4 promoter activity, suggesting the transactivation abilities of p53-WT are required for NOX4 repression (Figure 7D). Conversely, mutating the transactivation domain of the tumor-associated mutants ( $\mathrm{p} 53-\mathrm{R} 175 \mathrm{H}$ and p53-D281G) did not affect NOX4 promoter activity. To determine if a functional p53-WT transactivation domain is required for binding the NOX4 promoter, we preformed ChIP assays and found that unlike p53-WT, p53-L22Q/ W23S did not precipitate with the -4729/-4644 region (Figure 7E). We also observed a significant loss of SMAD3 recruitment in cells overexpressing p53-L22Q/W23S, suggesting p53-WT and SMAD3 form a repressor complex inhibiting NOX4 expression.

\section{Histone deacetylase (HDAC) activity is involved in p53-WT-mediated repression of NOX4 mRNA expression and promoter activity}

Previous studies demonstrated p53-WT can recruit HDACs to deacetylate histones associated with the promoters of genes involved in cell survival and migration $[18,30]$. HDACs catalyze removal of acetyl groups from histone lysine residues, thereby inhibiting transcription factor binding and gene expression [31]. We examined the possibility of HDACs participating in p53-WT-based repression of NOX4. We found NOX4 mRNA expression was relieved of $\mathrm{p} 53-\mathrm{WT}$ repression in Hep3B cells treated with Scriptaid, an HDAC inhibitor, for 24 hours compared to untreated cells (Figure 8A). Interestingly, we observed an increase in NOX4 mRNA expression in the vector-transfected cells treated with 

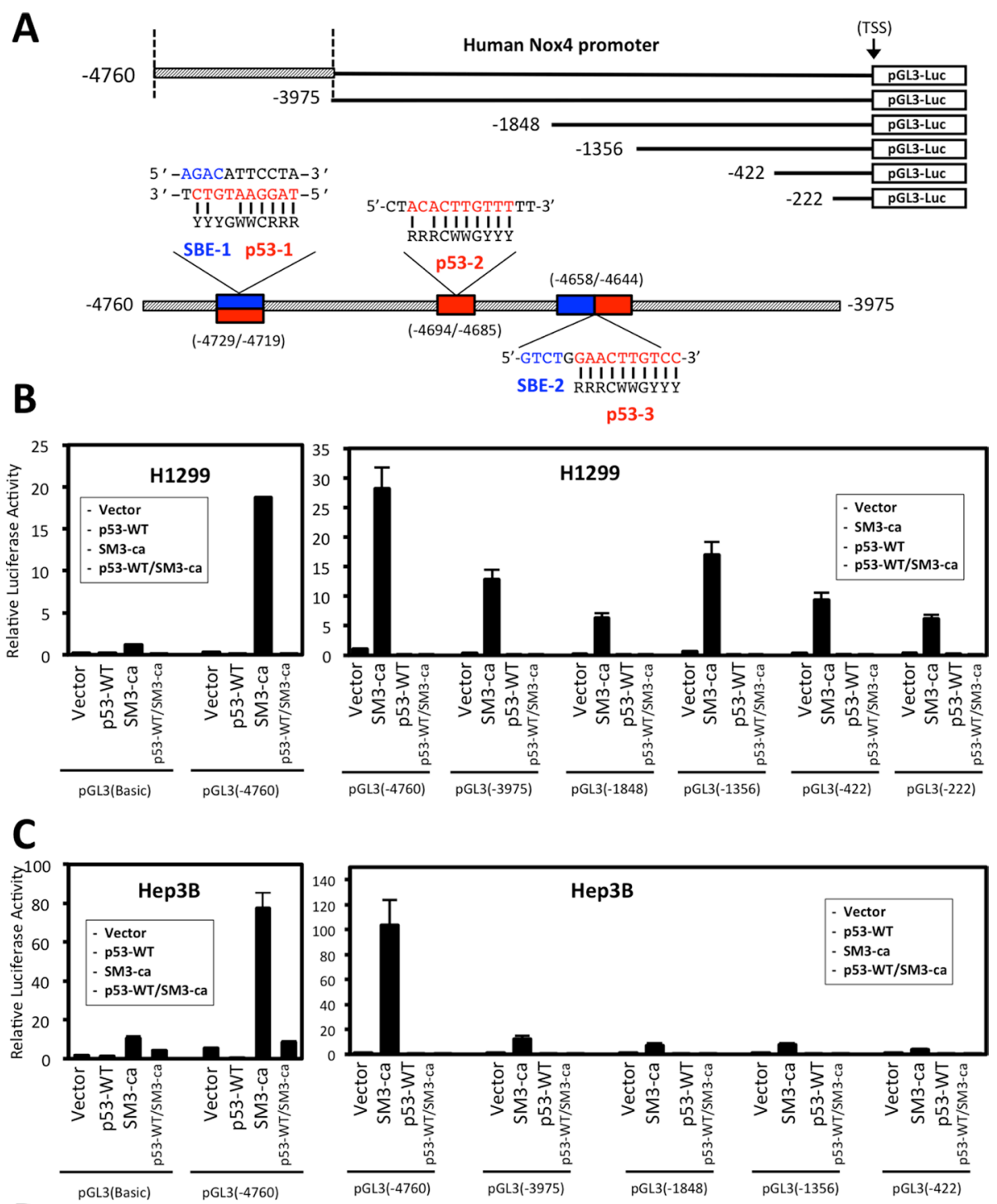

D

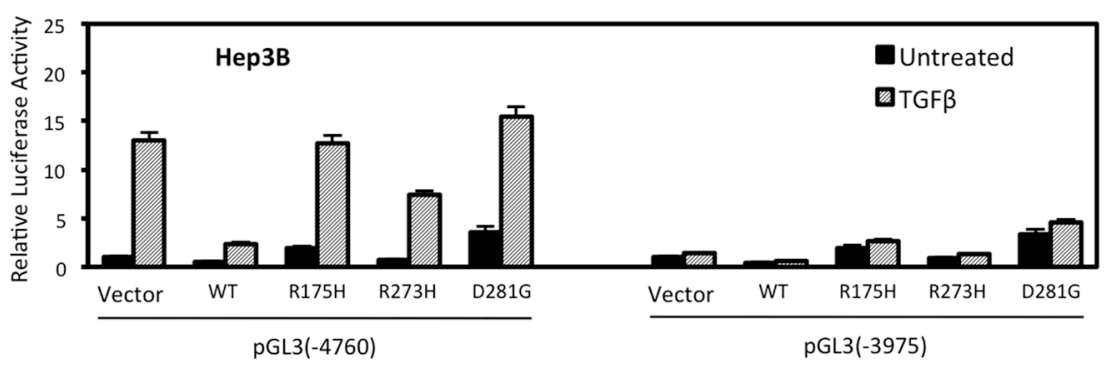

Figure 5: Deletion analysis of the NOX4 promoter reveals TGFß/SMAD3 and p53 regulatory sequences. (A) Schematic of 5'-end deletion series of the human NOX4 promoter starting from the transcription start site (TSS) was cloned into the pGL3-luciferase reporter vector to measure promoter activity. Putative SMAD binding elements (SBE) and p53 response elements are indicated within the -4760/-3975 promoter regions. The traditional p53 response element (p53-RE) consensus sequence consists of two 10-base decamers with a 0 -13-base spacer: RRRCWWGYYY ...n $=0-13$ bp...RRRCWWGYYY ( $\mathrm{R}$ is a purine $(\mathrm{A} / \mathrm{G}), \mathrm{Y}$ is a pyrimidine $(\mathrm{C} / \mathrm{T})$, and $\mathrm{W}$ is (A/T) [27]. However, studies have shown that p53 target genes that have one of the two 10-base decamers, or "half site" within the promoter can still be regulated by $\mathrm{p} 53$ binding $[26,27]$. The consensus sequences for SMAD binding elements (SBE) are CAGA and its reverse complement GTCT.[41] Above SBE are indicated in blue and p53-RE in red. (B) H1299 cells and (C) Hep3B cells were co-transfected with designated pGL3-Basic control or NOX4 promoter reporter plasmids and either vector control, SMAD3ca, p53-WT, or both p53-WT/ SMAD3ca plasmids. Luciferase activity was determined 48 hours after transfection $(n=3$, in triplicate). (D) Hep3B cells co-transfected with NOX4 promoter pGL3 (-4760) or pGL3 (-3975) and either vector control, p53-WT, p53-R175H, p53-R273H, or p53-D281G plasmids for 24 hours. The cells were then left untreated or treated with TGF $(5 \mathrm{ng} / \mathrm{ml})$ for an additional 24 hours. Total cell lysates were collected following TGF $\beta$ treatment and analyzed for luciferase activity $(n=3$, in triplicate). 
Scriptaid, indictaing inhibition of HDACs also enhances basal expression of NOX4. Moreover, we observed a slight increase in NOX4 protein expression in response to HDAC inhibition indicating HDACs regulate NOX4 primarily at the transcriptional level resulting in increased NOX4 protein levels (Figure 8B). Furthermore, p53-WT repression of NOX4 promoter activity was lost upon treatment of H1299 or Hep3B cells with either Scriptaid or trichostatin A (TSA) HDAC inhibitors (Figure 8C, 8D). H1299 and Hep3B cells transfected with NOX4 promoter reporter treated with HDAC inhibitors relieved significant amount of basal promoter repression, indicating that histone deacetylation has a role in repressing NOX4 even in the absence of p53-WT.

\section{p300 histone acetyltransferase (HAT) activity enhances mutant-p53-mediated NOX4 promoter activity and cell migration}

Next, we investigated the possibility of histone acetyltransferase (HAT) involvement in p53-mediated NOX4 expression. Studies have indicated tumorassociated p53 mutants affect histone modifications and transcriptional co-activation of multiple pro-oncogenic and pro-migratory genes $[17,20]$. Moreover, mut-p53 was shown to interact with p300, a transcriptional coactivator and histone acetyltransferase, and promote the transcription of cell cycle regulatory genes [20]. When we co-transfected Hep3B cells with p300 and the NOX4 (-4760) promoter reporter, we observed a significant gain in TGF $\beta$-induced promoter activity in control cells and in cells expressing p53-R175H or p53-D281G, but not p53-WT (Figure 9A). Conversely, co-expression of the HAT-inactive p300 mutant (p300دHAT) with p53$\mathrm{R} 175 \mathrm{H}$ or $\mathrm{p} 53-\mathrm{D} 281 \mathrm{G}$ resulted in reduction of TGF $\beta$ induced promoter activity relative to p300 co-expression, indicating HAT activity is involved in mut-p53-mediated NOX4 promoter activation. Interestingly, we found that HAT activity was not involved in TGF $\beta$-induced promoter activity in control cells, suggesting p300 HAT activity is utilized specifically in mut-p53-dependent regulation of the NOX4 promoter.

Next, we examined the status of promoter histone acetylation by ChIP assay using an antibody specific for acetylated histone-4 lysine-8 (H4K8). Previous reports have shown that $\mathrm{p} 300$-mediated acetylation of H4K8 is
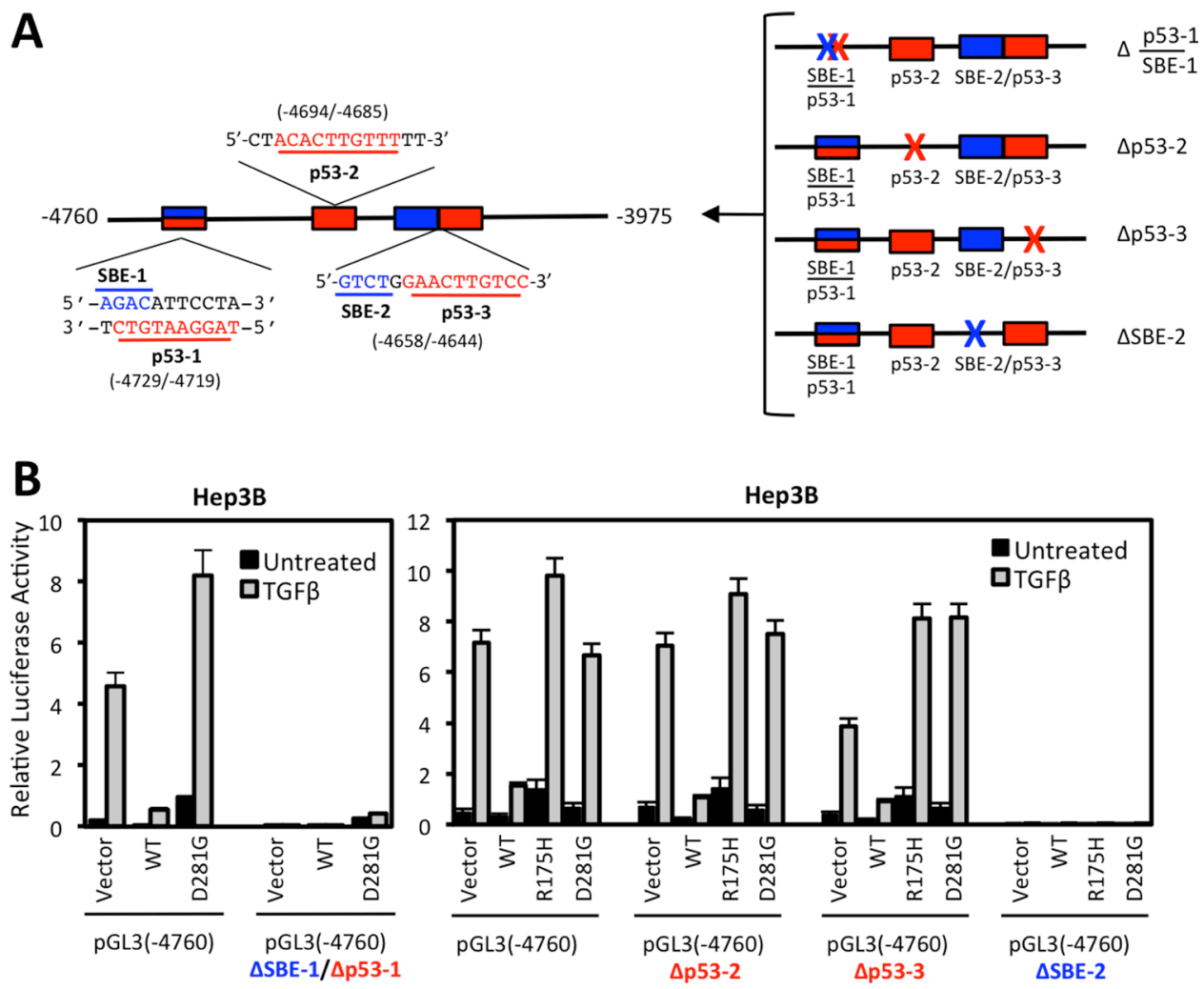

Figure 6: Deletion of conserved SMAD binding elements (SBE) or p53 response elements (p53-RE) reduce NOX4 induction by TGFß. (A) Schematic of the SBE (blue) and p53 response element (red) sequences and their position within the (-4760) to (-3975) segment of the NOX4 promoter (left panel). SBE (blue X) or p53RE (red X) sequences were deleted in the pGL3-NOX4 promoter-reporter (-4760) to determine functionality (right panel). (B) Hep3B cells were co-transfected with designated pGL3-NOX4 promoter-reporter vectors and either vector control, p53-WT, p53-R175H, or p53-D281G plasmids for 24 hours. Transfected cells were either left untreated or treated with TGF $\beta(5 \mathrm{ng} / \mathrm{ml})$ for an additional 24 hours. Promoter activity was determined by luciferase assay $(n=3$, in triplicate). 
associated with transcriptional activation [32]. We found TGF $\beta$ treatment increased H4K8 acetylation associated with the NOX4 promoter which was augmented by p53-D281G expression (Figure 9B). Conversely, p53WT expression severely blunted H4K8 acetylation, suggesting histone modifications play a significant role in the divergent effects of mut-p53 and p53-WT on TGF $\beta$ mediated NOX4 expression.
Thus far, our results indicate p300 and mutant p53 co-regulate the NOX4 promoter. These findings prompted us to investigate whether p300 and mutant p53 also have roles in basal NOX4 expression. To do this, we depleted the endogenous expression of p53-R273H, p300, or both by siRNA-mediated knockdown in PANC-1 pancreatic tumor cells. Knock down of either p53-R273H or p300 resulted in a reduction of basal NOX4 protein expression compared

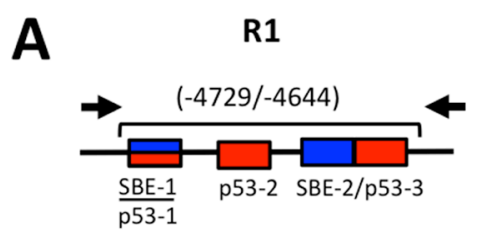

B

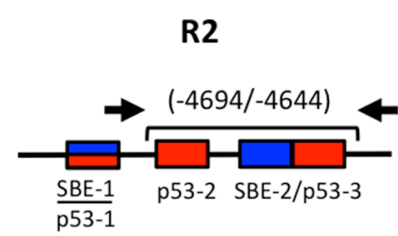

C

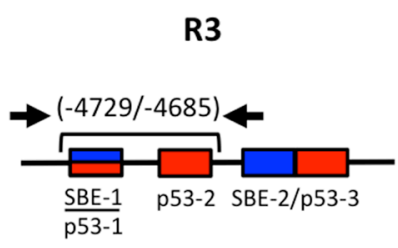

D

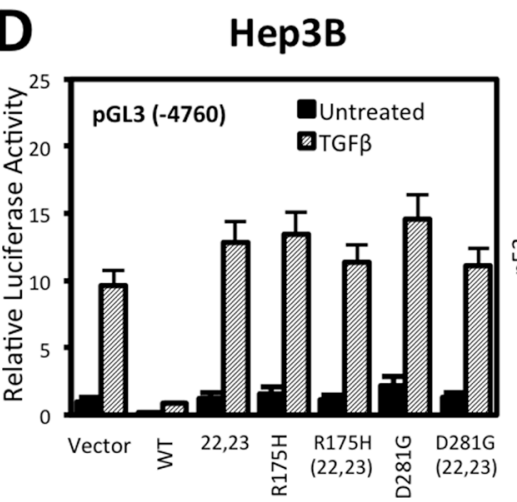

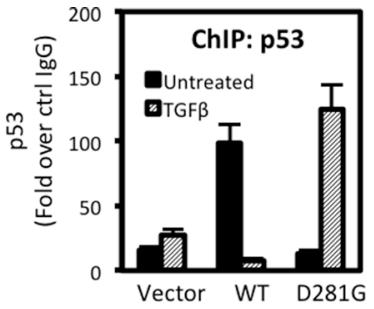
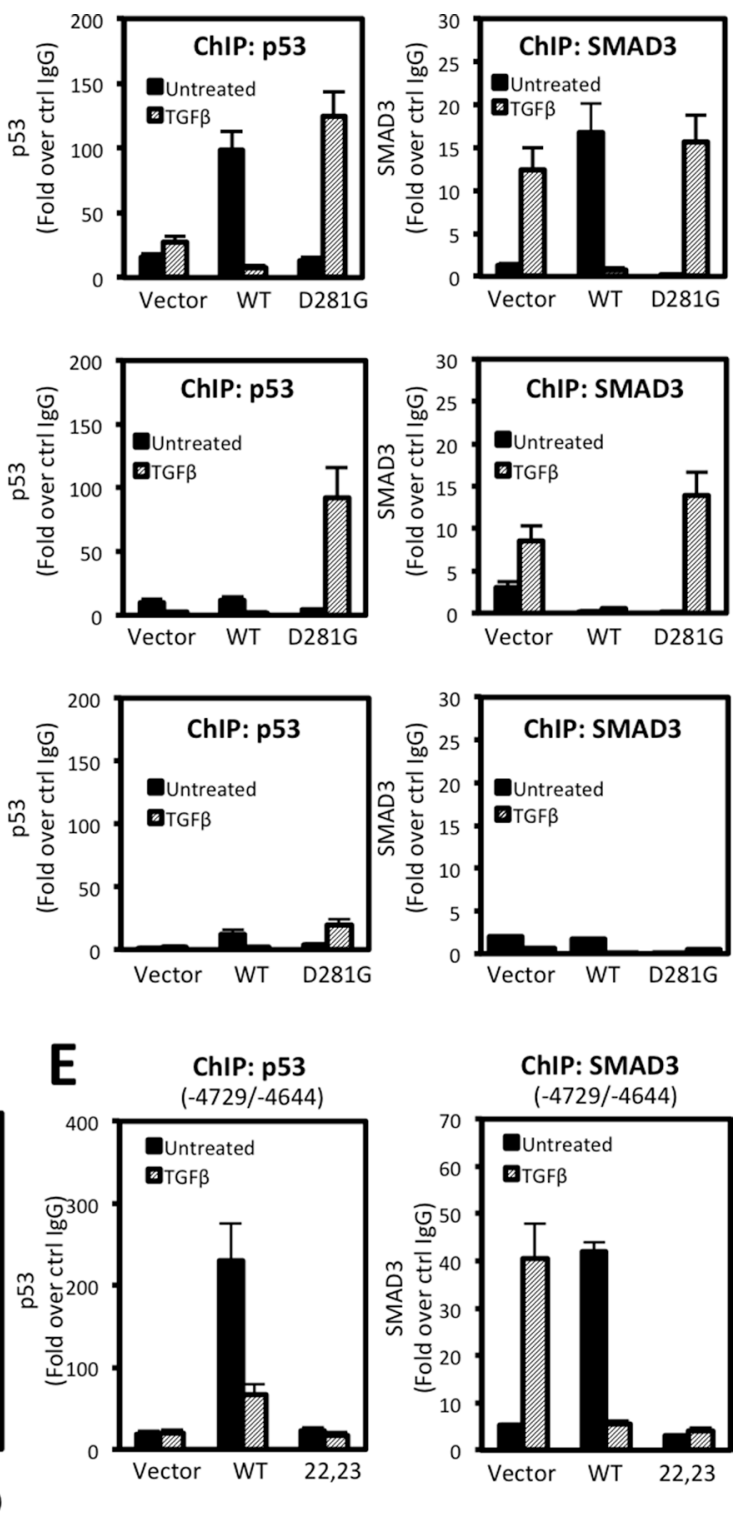

Figure 7: p53 and SMAD3 associate with NOX4 SBE and p53RE sequences in a TGFß-dependent manner. (A-C) Schematic showing the NOX4 promoter and the p53 and SMAD target regions tested in ChIP assays (left). See Materials and Methods for primer sequences. ChIP was performed in Hep3B cells transfected with vector control, p53-WT, or p53-D281G plasmids for 24 hours and subsequently treated with TGF $\beta(5 \mathrm{ng} / \mathrm{ml})$ for an additional 24 hours. ChIP assays were performed using antibodies that specifically recognize p53, SMAD3, or IgG (negative control). Input DNA and immunoprecipitated DNA were quantified by qPCR. The ChIP-qPCR results are represented as fold enrichment of IP with anti-p53 (middle) or anti-SMAD3 (right) ChIP over input DNA relative to IgG negative control $(n=3)$. (D) Luciferase assays were performed in Hep3B cells using the pGL3-NOX4 promoter-reporter (-4760). The cells were transfected with control vector, p53-WT, p53-L22Q/W23S (transactivation domain mutant), mut-p53-R175H, p53-D281G, or the triple mut-p53-R175H/L22Q/W23S or p53-D281G/L22Q/W23S. Twenty-four hours after transfection, cells were left untreated or treated with TGF $\beta(5 \mathrm{ng} / \mathrm{ml})$ for an additional 24 hours. (E) ChIP assay was performed as described in panel A in Hep3B cells that were expressing control, p53-WT, or p53-L22Q/W23S followed by 24 hours stimulation with TGF $\beta(5 \mathrm{ng} / \mathrm{ml})$ ( $n=2$, in duplicate). 


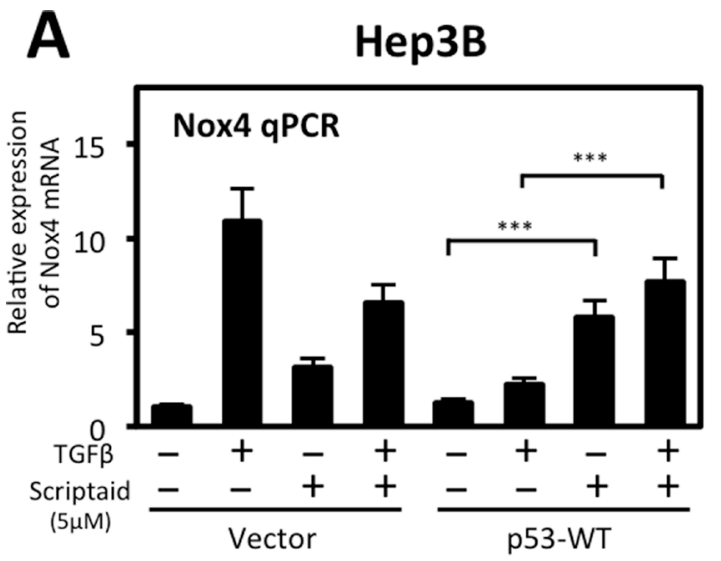

B

Hep3B
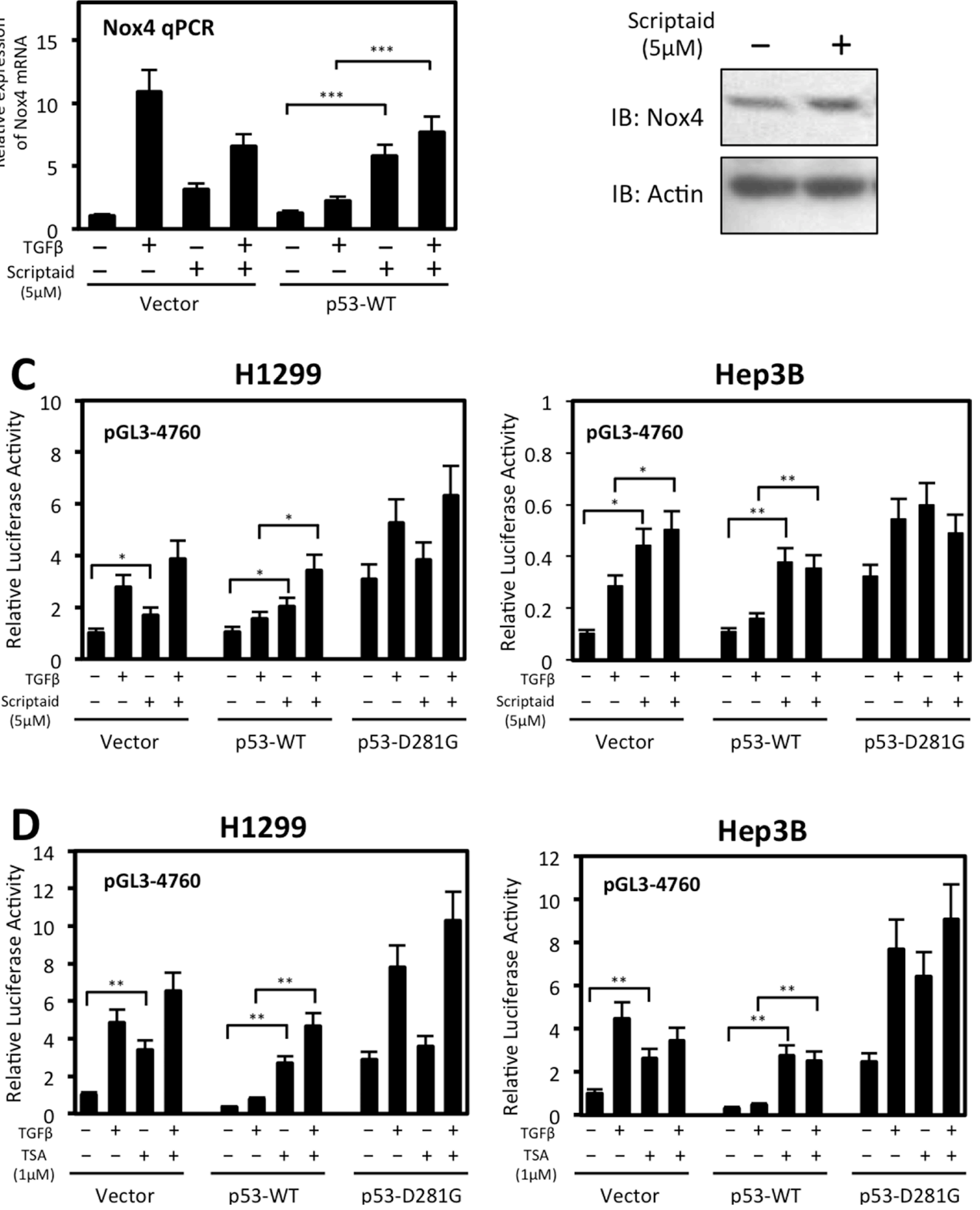

Figure 8: Histone deacetylase (HDAC) activity participates in wild-type p53-mediated repression of NOX4 mRNA and promoter activity. (A) Hep3B cells were transiently transfected with control vector or p53-WT. Twenty-four hours after transfection, cells were treated with TGF $\beta(5 \mathrm{ng} / \mathrm{ml})$ and either Scriptaid $(5 \mu \mathrm{M})$ or DMSO for 24 hours. Real-time qPCR analysis of NOX4 mRNA expression was detected with NOX4-specific primers. The relative mRNA level was normalized to GAPDH control. $(n=4)(\mathbf{B})$ Hep3B cells transfected with empty vector were treated with Scriptaid $(5 \mu \mathrm{M})$ for 24 hours. After 24 hours, total cell lysates were collected and analyzed by immunoblot. (C) H1299 or Hep3B cells were co-transfected with pGL3-NOX4 (-4760) and either vector control, p53-WT, or p53-D281G. Twenty-four hours later, cells were left untreated or treated with TGF $\beta(5 \mathrm{ng} / \mathrm{ml})$ and either Scriptaid $(5 \mu \mathrm{M})$ or DMSO (-) for another 24 hours. Total cell lysates were then collected and assayed for luciferase activity. (D) Luciferase assays were conducted on H1299 or Hep3B cells co-transfected with pGL3-NOX4 (-4760) p53 plasmids as in B. Twenty-four hours later, cells were left untreated or treated with TGF $\beta(5 \mathrm{ng} / \mathrm{ml})$ and either trichostatin A (TSA) $(1 \mu \mathrm{M})$ or DMSO (-) for 24 hours $(n=3$, in triplicate). Significance values are indicated as $* P$-value $<0.05, * * P$-value $<0.01$, or $* * * P$-value $<0.001$. 
to control (Figure 9C). Collectively, these observations indicate p300 related epigenetic mechanisms are involved in mutant p53-dependent induction of Nox4.

We demonstrated in previous work that mut-p53 and NOX4 are necessary for TGF $\beta$-mediated cell signaling and migration of different human tumor cell lines [21]. A recent study demonstrated p300 involvement in pancreatic tumor cell migration and invasion [33]. Therefore, we investigated whether p300 is involved in mut-p53/NOX4-dependent cell migration. We conducted scratch wound repair assays in 96-well plates using the 96-pin WoundMaker instrument to create 96 reproducibly uniform wounds approximately $800 \mu \mathrm{m}$ in width. Wound assays conducted on $\mathrm{H} 1299$ cells demonstrated that coexpression of p53-D281G and p300 increased TGF $\beta$ induced wound closure (Figure 9C). However, this increase in cell migration was reduced in cells co-transfected with dominant negative NOX4-P437H, confirming that NOX4 has a significant role in TGF 3 -stimulated, p53-D281G/ p300-mediated cell migration. Furthermore, we found that p300 HAT activity is critical in p300/p53-D281G-mediated wound closure, which corroborates our findings in Figure 8A showing that $\mathrm{p} 300$ with HAT activity supports TGF $\beta /$ mut-p53 induction of NOX4 promoter activity. In contrast, p53-WT alone or co-expressed with p300 resulted in a reduction in TGF $\beta$-dependent wound closure.

\section{DISCUSSION}

Previously, we described NOX4 as a TGF $\beta /$ SMAD3inducible source of ROS in both normal and metastatic epithelial cells $[34,35]$. We provided strong evidence of NOX4 having an important role in common EMTrelated events including elevated cellular ROS production, increased fibronectin expression, and increased cell migration and invasion [34]. We also reported that the mutational status of p53 is an important determinant of TGF $\beta / S M A D 3-m e d i a t e d$ NOX4 regulation in breast and lung epithelial cells, showing that p53- WT is a suppressor of NOX4 induction whereas tumor-associated mutant forms of $\mathrm{p} 53$ enhance NOX4 expression and ROS generation [21]. Consequently, mut-p53-induced NOX4 results in increased focal adhesion kinase (FAK) activation and cell motility [21]. Here, we confirmed and extended these findings on differential regulation of NOX4 by mutant and $\mathrm{p} 53$-WT based on primary tumor expression data from TCGA and examined the effects of several common tumorassociated p53 mutants on NOX4 mRNA expression and promoter activity as well as tumor cell migration.

Our study provides insight into the mechanisms of p53 and SMAD3 transcriptional regulation of NOX4. We identified critical SBE and p53RE required for NOX4 induction by mut-p53 within the $-4760 /-3975$ bp region upstream of the transcription start site of NOX4. Previously, Bai et al. identified an AP-1/SMAD box critical for transcriptional activation of NOX4 by TGF $\beta$ in lung fibroblasts [22]. This finding corroborates with our studies by deletion analysis of SBE-2 and by ChIP binding assays using SMAD3-specific antibodies. ChIP analysis of TGF $\beta$ treated Hep3B cells revealed SMAD3 and p53-D281G are associated with the NOX4 promoter, whereas SMAD3 and p53-WT associate only in the absence of TGF $\beta$. Furthermore, disruption of the transactivation domain of p53-WT (L22Q/W23S) caused a loss of its repressive effect on TGF $\beta$-induced NOX4 promoter activity. In support of this, ChIP assays revealed a substantial loss of both SMAD3 and p53-L22Q/W23S binding to the NOX4 promoter, suggesting the transactivation domain is a required for SMAD3 associations with p53-WT as a negative co-regulator. However, it is worth noting that the distance of mut-p53 and SMAD3 binding (-4729/4644 bp) from the transcription start site suggests these factors are associated with an enhancer-like region or complex to regulate NOX4 transcription.

One mode of transcriptional repression by $\mathrm{p} 53$ is the recruitment of co-repressors and chromatin-modifying enzymes such as HDACs [36]. Histone acetylation is balanced by the activities of acetyltransferases and deacetylases. Acetyltransferases are associated with increased gene transcription and deacetylases with repressed gene expression [37]. Here, we show inhibition of HDACs abolished p53-WT repression of NOX4 promoter activity and mRNA expression, suggesting HDACs play a role in p53-WT-mediated NOX4 repression. Conversely, overexpression of p300 enhanced mut-p53-mediated NOX4 promoter activity, whereas the HAT-inactive p300 reduces these effects. Moreover, we observed a TGF $\beta$ induced increase in acetylaytion of histones associated with the NOX4 promoter, which was augmented by p53-D281G. We also showed expression of an inactive mutant from of NOX4-P437H attenuated p53-D281G/p300-mediated cell migration stimulated by TGF $\beta$. Here we provided further support for the involvement of p300 as a mediator of NOX4-dependent cell migration. The acetylation of histones associated with the NOX4 promoter by p300 may be an important epigenetic marker in determining the progression of tumors expressing mut-p53.

Together, our data suggest mut-p53 enhances TGF $\beta /$ SMAD3-driven NOX4 promoter activity involving histone modifications mediated by $\mathrm{p} 300$. In contrast, $\mathrm{p} 53$-WT requires a functional transactivation domain to exert its repressive effect on NOX4, suggesting p53-WT associates with other repressive co-factors. Transcriptional repression of NOX4 by p53-WT is also in part due to HDAC enzyme activity (Figure 10). Further investigation is necessary to establish additional co-factors involved in transcriptional regulation of NOX4 by both p53-WT and mut-p53. Our previous and current findings confirm a correlation between tumor-associated p53 mutations and increased NOX4 expression and downstream events promoting tumor cell migration, suggesting NOX4 is an attractive therapeutic target to abolish or decrease metastatic progression in multiple cancer types. 

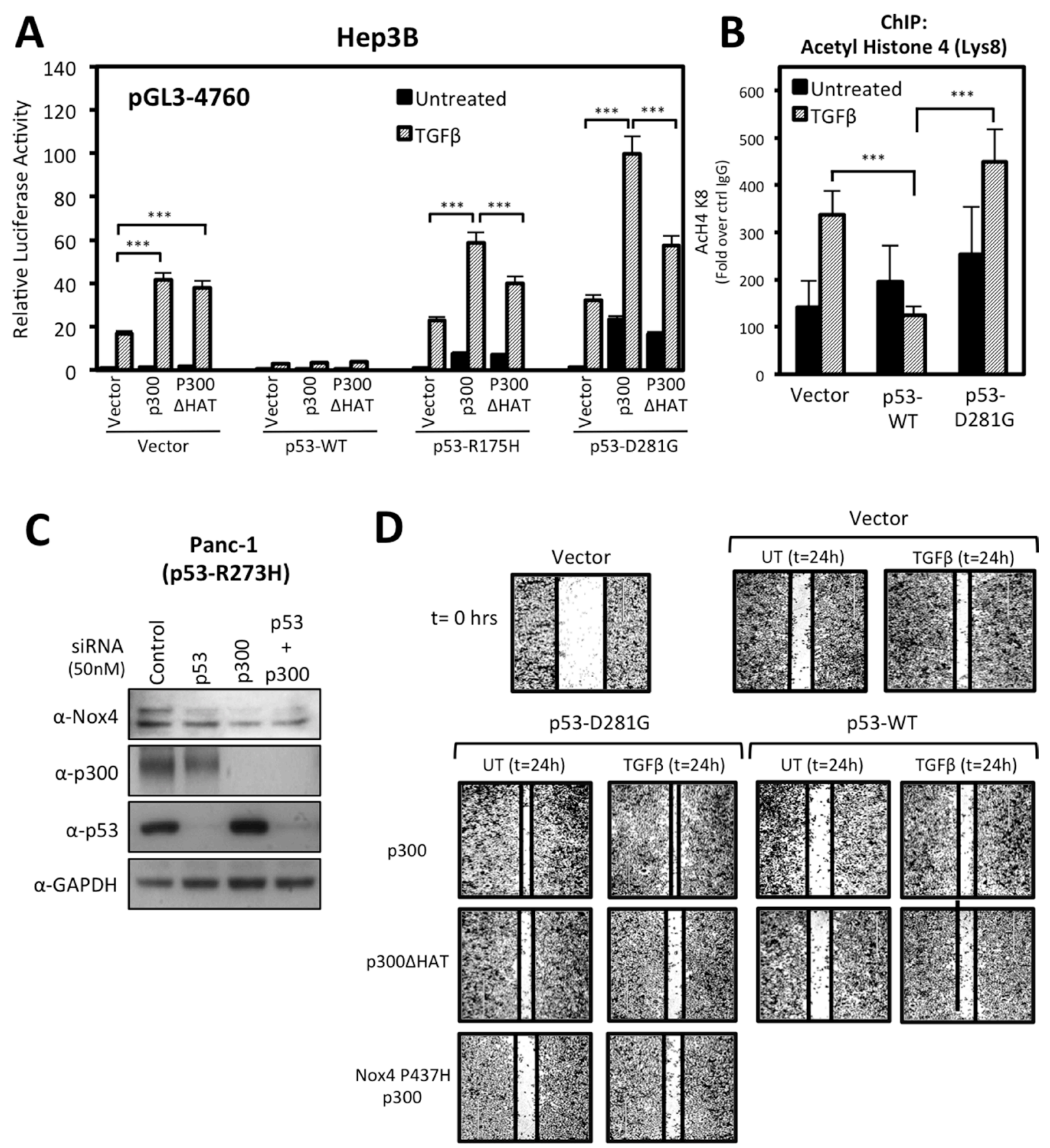

Figure 9: p300 histone acetyltransferase (HAT) activity enhances mutant p53-mediated NOX4 promoter activity and cell migration. (A) Hep3B cells co-transfected with NOX4 promoter pGL3 (-4760) and either vector control, p53-WT, p53-R175H, or p53-D281 G; and either control, p300, or HAT-inactive p300 $\mathrm{HAT}$ plasmids for 24 hours. The cells were then treated with TGF $\beta(5 \mathrm{ng} / \mathrm{ml})$ for an additional 24 hours. Total cell lysates were collected following TGF $\beta$ treatment and analyzed for luciferase activity $(n=3$, in triplicate). (B) ChIP assays were performed in Hep3B cells expressing control, p53-WT, or p53-D281G plasmids and either treated with TGF $\beta(5 \mathrm{ng} / \mathrm{ml})$ for 24 hours or remained untreated. Histone immunoprecipitation was conducted using ChIP qualified antibodies specific for acetylated lysine-8 histone 4 (H4K8) antibodies, or IgG (negative control). ChIP-qPCR results are represented as fold enrichment of IP: $\alpha-\mathrm{H} 4 \mathrm{~K} 8 \mathrm{ChIP}$ over input DNA relative to IgG negative control $\left(n=2\right.$, in triplicate). (C) PANC-1 ${ }^{(553-\mathrm{R} 273 \mathrm{H})}$ cells were transfected with On-Target SMARTpool p53-specific siRNAs $(50 \mathrm{nM})$, p300-specific siRNAs $(50 \mathrm{nM})$, or non-targeting control siRNAs $(50 \mathrm{nM})$ for $72 \mathrm{~h}$. Fifty micrograms of total cell lysates were analyzed by immunoblotting. (D) H1299 cells transfected with vector control, p53-WT, p53D281G, p300-WT, p300- $\triangle$ HAT, or NOX4-P437H plasmids and seeded into a 96-well tissue culture plate $\left(3.5 \times 10^{4}\right.$ per well $)$ and grown to confluence. Wounds were made using the WoundMaker 96-pin tool, which creates 96 precise and reproducible wounds of $800 \mu \mathrm{m}$. The cells were then left untreated or were treated with TGF $\beta(5 \mathrm{ng} / \mathrm{ml})$. Approximately $18-22$ hours after wounding and TGF $\beta$ treatment, the cells were fixed and stained for imaging. The images presented are representative of four experiments completed in triplicate. Significance values are indicated as $* * * P$-value $<0.001$. 

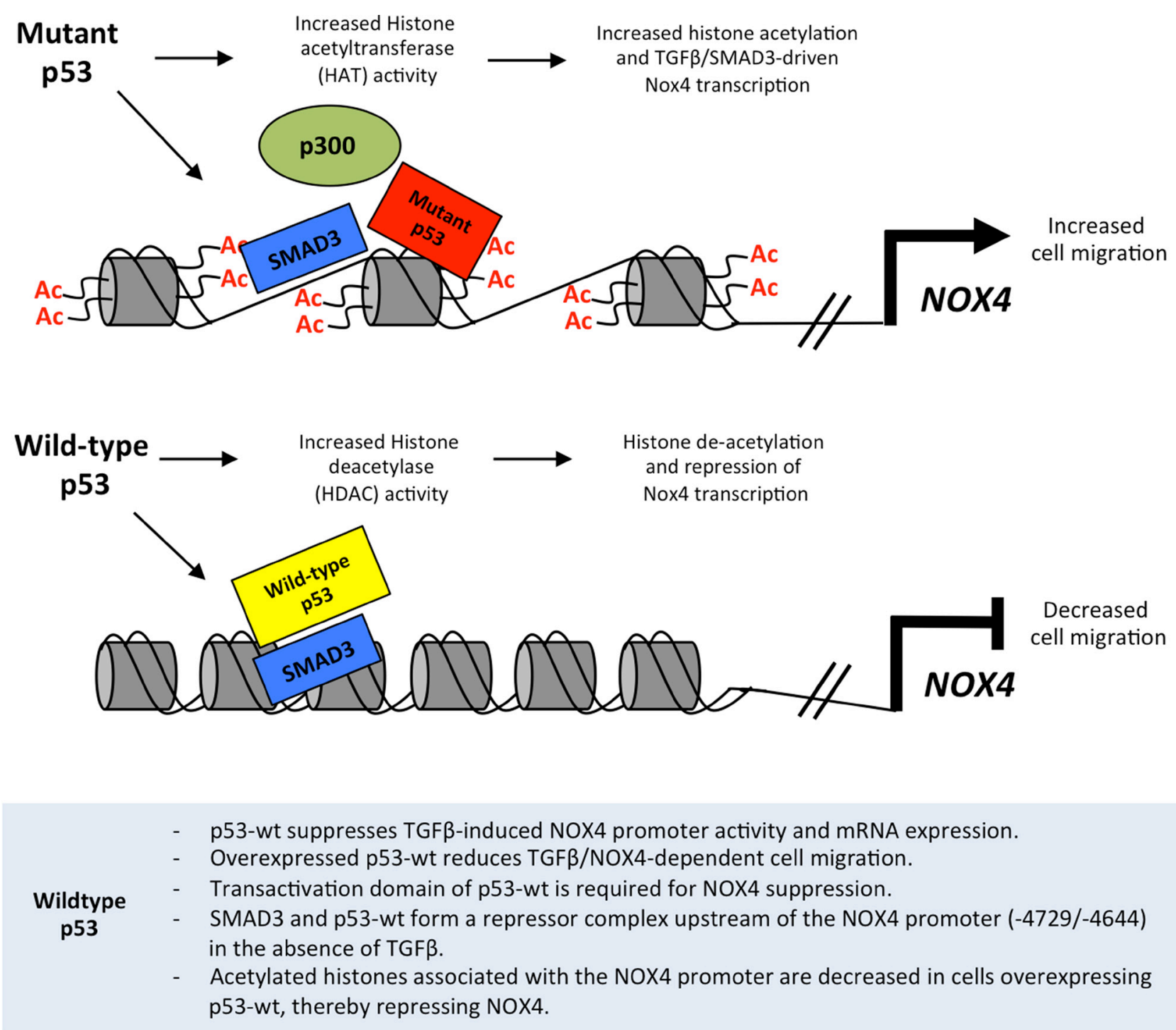

Mutant
p53

p53-mt silencing reduces NOX4 expression (Panc-1 (R273H) and PLC/PRF5 (R249S) cells). Overexpressed mut-p53 (R175H, R249S, R273H, D281G) supports NOX4 induction by TGF 3 . - p53-D281G increases NOX4 promoter-associated histone acetylation and cell migration. - p53-D281G and SMAD3 form a complex upstream of the Nox4 promoter (-4694/-4644).
- p300 HAT activity augments TGF $3 /$ mut-p53 (R175H and D281G)-induced promoter activity. p300 silencing reduces NOX4 protein expression (Panc-1 cells).
- Inactivation of p300 HAT activity reduces TGF $3 /$ mut-p53 (D281G)-mediated cell migration.

HDAC - Tricostatin A (TSA) and Scriptaid relieve repressive effects of p53-wt on the NOX4 promoter inhibitors and mRNA expression, indicating HDACs participate in p53-wt-mediated NOX4 repression.

Figure 10: Model of the divergent roles of wild-type and mutant p53 regulating NOX4 expression and cell migration through histone modifications. Mut-p53 and SMAD3 co-localize to SBE and p53-RE sequences in the NOX4 gene. Mutant p53 supports p300-mediated acetylation of histones associated with NOX4 regulatory sequences, which enhances TGF $\beta / S M A D 3$-driven NOX4 promoter activity and subsequent NOX4-dependent migration of tumor cells. In contrast, p53-WT and SMAD3 co-localize to SBE and p53-RE sequences to suppress NOX4. Histone deacetylases are recruited by p53-WT to the NOX4 locus, thereby repressing NOX4. Summary (below) outlines experimental evidence from the current study supporting the schematic model. 


\section{MATERIALS AND METHODS}

\section{The cancer genome atlas (TCGA) analysis}

To identify whether p53 mutation status correlates with NOX4 mRNA expression, we extracted expression data as z-score values and mutation status from TCGA via cBioPortal [38, 39]. We performed Mann-Whitney Wilcoxon tests where appropriate to determine significant differences in NOX4 expression levels between mut-p 53 and p53-WT. We also screened for possible relationship between NOX4 and fibronectin mRNA expression. We extracted fibronectin data from TCGA and employed Spearman rank correlation analysis as well as simple linear regression of the $\log _{\mathrm{e}}$-transformed data to detect relationships between NOX4 and fibronectin mRNA expression. Statistical analyses were performed in R [40]. Samples with extreme outliers were not included in final plots but were included in the statistical analysis. The R script and copies of data used are available at github.com/wfma/HEBoudreau.

\section{Cell culture}

Human cell lines H1299, PCL/PRF/5, HepG2, PANC-1, and Hep3B were from ATCC (Manassas, VA, USA). H1299 cells were maintained in RPMI (Thermo Fisher, Waltham, MA, USA) with $10 \%$ fetal bovine serum (FBS) (Thermo Fisher) and $100 \mathrm{mg} / \mathrm{ml}$ of penicillinstreptomycin. HepG2, Hep3B and PANC-1 cells were maintained in DMEM (Thermo Fisher) with $10 \%$ FBS and $100 \mathrm{mg} / \mathrm{ml}$ of penicillin-streptomycin. PLC/PRF/5 cells were maintained in MEM- $\alpha$ (Thermo Fisher) with $10 \%$ FBS and $100 \mathrm{mg} / \mathrm{ml}$ of penicillin-streptomycin. Cells were grown in a humidified atmosphere of $5 \% \mathrm{CO}_{2}$ and $95 \%$ air at $37^{\circ} \mathrm{C}$. The following reagents were used: TGF $\beta-1$ (Peprotech, Rocky Hill, NJ, USA); TGF $\beta$ receptor I-specific inhibitor 616451 (EMD Millipore, Billerica, MA, USA); SMAD3-specific inhibitor SIS3 (SigmaAldrich, St. Louis, MO, USA); Scriptaid, Trichostatin A, and 5-fluorouracil were from Sigma-Aldrich.

\section{Plasmids}

pCMV-p53-R175H， p53-R248Q， p53-R249S, p53-R273H，p53-R280K，p53-D281G were generated as previously described [21]. pcDNA3.1-p300 and pcDNA3.1-p300(HAT-) were from Warner Greene (Addgene \#23252 and \#23254). The pGL2-p21 promoterLuc was from Martin Walsh (Addgene \#33021). The NOX4-pGL3 luciferase reporter plasmids -4760 and -3975 were provided by Victor Thannickal (University of Alabama) [22]. Other NOX4-pGL3 promoter reporter plasmids were generated as previously described [35]. The pGL3-Basic control vector was from Promega (Promega Corporation, Madison, WI, USA). TGFßRI (T204D) was previously described [34]. SMAD2 and SMAD3 were from Lalage Wakefield (NIH/NCI, Bethesda, MD). Active SMAD2 and SMAD3 were pseudo-phosphorylated at serine residues (SMAD2: serine residues 464, 465, and 467) (SMAD3: serine residues 422, 423, and 425) by replacement with aspartic acid residues.

\section{Transient transfections}

Hep3B, PCL/PRF/5, PANC-1, and H1299 were transfected with Fugene 6 (Promega, Madison, WI, USA) using manufacturer's protocols. H1299 cells, for migration, studies were transfected with Lipofectamine 3000 (Thermo Fisher) according to the manufacturer's protocols.

\section{siRNA-mediated gene knockdown}

Dharmacon ON-TARGETplus SMARTpool siRNAs were used for silencing endogenous p53 (TP53 siRNAs L-003329-00-0005) and p300 (EP300 siRNAs L-00348600-0005) (Dharmacon, Lafayette, CO, USA). Non-targeting siRNAs (D-001810-01-05) were used as the control. DharmaFECT4 reagent was used for siRNA transfections according to manufacturer's protocols. The cells were transfected in antibiotic-free medium at a concentration of $50 \mathrm{nM}$ for 72 hours.

\section{RNA isolation and cDNA synthesis}

Total RNA from cells was extracted with Trizol (Thermo Fisher). One microgram of total RNA was used for Thermoscript (Thermo Fisher) RT-PCR. Both were conducted according to manufacturer's protocols.

\section{Quantitative PCR}

Gene expression was quantified by qPCR using an ABI Prism 7500 RT-PCR System (Applied Biosystems, Foster City, CA, USA). Cellular RNA was reverse transcribed with ThermoScript. SYBR Green PCR mix (Thermo Fisher) was used to detect mRNA expression with the following human specific primers: (NOX4) forward: 5'-TGAACTATGACCTCAGCCTCTGCG-3', reverse: 5'-ATGACTGGAAACCATACAAGCT-3, (p21/CDKN1A) forward: 5'-CCGAAGTCAGTTCCTTGTGG-3', reverse: 5'-CATGGGTTCTGACGGACAT $-3^{\prime}$ and (GAPDH) forward: 5'-AGCCACATCGCTCAGACAC-3', reverse: 5'-GCCCAATACGACCAAATCC-3'. GAPDH was used as an internal control for normalization. The qPCR results are represented as relative quantification $(\mathrm{RQ})$, which is $\mathrm{RQ}=$ $2^{(-\Delta \Delta C t)}$. The RQ value is the fold change in gene expression compared to the control sample.

\section{Luciferase assay}

Luciferase assays were performed according to manufacturer's protocols (Promega). Briefly, $1 \times 10^{5}$ cells 
were seeded per well in 12-well dishes. Each well was transfected with $0.5 \mu \mathrm{g}$ of pGL3-NOX4 promoter reporter plasmid 24 hours later. Luciferase expression was assayed 48 hours later with Luminoskan luminometer (Thermo Fisher).

\section{Chromatin immunoprecipitation (ChIP)}

Cells were cultured on $150 \mathrm{~mm}$ and fixed with $1 \%$ formaldehyde (10 min, RT), followed by $200 \mathrm{mM}$ Glycine for $10 \mathrm{~min}$. The cells were washed twice with ice-cold 1 $\mathrm{X}$ PBS, and collected in lysis buffer $(1 \times$ PBS containing protease inhibitor cocktail (PIC) and $0.5 \mathrm{mM}$ PMSF). Chromatin preparations were sheared using ChIP-IT Express Enzymatic Shearing Kit according to manufacturer's protocols (Active Motif, Carlsbad, CA, USA). Immunoprecipitations were performed using Magna ChIP A/G Kit according to manufacturer's protocol (Millipore). The following antibodies were used for immunoprecipitation: anti-SMAD3 (E.980.9) (Thermo Fisher); anti-acetyl histone H4 (Lys8) (Millipore); anti-p53 (DO-1); mouse IgG (Santa Cruz, Dallas, TX, USA). Quantitative PCR was used to quantify the immunoprecipitated DNA with the following NOX4 promoter-specific primers: (R1) forward: 5'- AAGGGCATAAGGACCTCTCC-3', reverse: 5'- AGGGAAAAGTGGTCCAAAG-3'; (R2) forward: 5'- CTGAATCAGATGATGGTCTACACTTG-3', reverse: 5'- GGTCCAAAGGCTTAACATTC-3'; (R3) forward: 5'- AAGGGCATAAGGACCTCTCC-3', reverse: 5'- GACTCATTCTCATTTCTAC-3'. The data are represented as fold change over $\mathrm{IgG}$ control antibody (Ct IP/Ct IgG). The $\mathrm{Ct}$ values were normalized to the input $(1 \%)$ of sheared chromatin DNA. The data are represented as fold change above background $=2^{\wedge}(-\Delta \Delta \mathrm{Ct})$.

\section{Western blotting}

Cell lysates were processed for Western blotting as previously described [21]. The following antibodies were used for immunoblotting: rabbit monoclonal anti-NOX4 (UOTR1B493) (Abcam, Cambridge, MA, USA); mouse monoclonal anti-p53 (DO-1) (Santa Cruz Biotechnology); rabbit monoclonal anti-phospho-SMAD3 (EP823Y) (Abcam); rabbit monoclonal anti-SMAD3 (EP568Y) (Abcam); and rabbit polyclonal anti-p300 (A300-358A) (Bethyl Laboratories, Montgomery, TX, USA).

\section{Cell migration assay}

H1299 cells were seeded on 6-well tissue culture plates $\left(4 \times 10^{5}\right.$ cells/ well) 24 hours before transfection. The cells were transfected with $2 \mu \mathrm{g}$ of vector control, p53-WT, p53-D281G, p300-WT, p300- $\Delta$ HAT, or NOX4$\mathrm{P} 437 \mathrm{H}$ plasmid DNA for 5 hours. The cells were then trypsinized and re-seeded into 96-well tissue culture plates $\left(3.5 \times 10^{4}\right.$ per well $)$ for monolayer woundhealing assays. Wounds were made using WoundMaker
(Essen Bioscience, Ann Arbor, MI, USA) 96-pin tool, creating reproducible uniform cell-free zones of $800 \mu \mathrm{m}$. Immediately following wounding, cells were washed twice then treated with TGF $\beta(5 \mathrm{ng} / \mathrm{ml})$ or left untreated. Approximately 18-22 hours after wounding and TGF $\beta$ treatment, the cells were fixed and stained using Diff Stain (IMEB Inc., San Marcos, CA, USA). Visible light images $(4 \times$ objective $)$ of fixed and stained migrating cells were captured with a phase contrast microscope (Evos FL Cell Imaging System, Life Technologies, Carlsbad, CA, USA).

\section{Statistical analysis}

Data are represented as means \pm s.d. of the results of at least three independent experiments. Student's $t$-test was used to calculate significant values, indicated as $* P$-value $<0.05, * * P$-value $<0.01$, or $* * * P$-value $<0.001$.

\section{Abbreviations}

Transforming growth factor- $\beta$ (TGF $\beta)$; NADPH oxidase-4 (NOX4); Reactive oxygen species (ROS); Histone acetyltransferase (HAT); Histone deacetylase (HDAC); SMAD binding element (SBE); p53 response element (p53RE).

\section{ACKNOWLEDGMENTS AND FUNDING}

This work was supported by funds from the Intramural Research Program of NIH, National Institute of Allergy and Infectious Diseases (ZO1-AI-000614). NOX4-pGL3 luciferase reporter plasmids -4760 and -3975 were a generously provided by Dr. Victor Thannickal (University of Alabama, Birmingham).

\section{CONFLICTS OF INTEREST}

The authors declare no conflicts of interest.

\section{REFERENCES}

1. Freed-Pastor WA, Prives C. Mutant p53: one name, many proteins. Genes Dev. 2012; 26:1268-1286.

2. Brosh R, Rotter V. When mutants gain new powers: news from the mutant p53 field. Nat Rev Cancer. 2009; 9:701-713.

3. Zhou G, Wang J, Zhao M, Xie TX, Tanaka N, Sano D, Patel AA, Ward AM, Sandulache VC, Jasser SA, Skinner HD, Fitzgerald AL, Osman AA, et al. Gain-offunction mutant $\mathrm{p} 53$ promotes cell growth and cancer cell metabolism via inhibition of AMPK activation. Mol Cell. 2014; 54:960-974.

4. Yeudall WA, Vaughan CA, Miyazaki H, Ramamoorthy M, Choi MY, Chapman CG, Wang H, Black E, Bulysheva AA, Deb SP, Windle B, Deb S. Gain-of-function mutant p53 
upregulates CXC chemokines and enhances cell migration. Carcinogenesis. 2012; 33:442-451.

5. Blandino G, Levine AJ, Oren M. Mutant p53 gain of function: differential effects of different p53 mutants on resistance of cultured cells to chemotherapy. Oncogene. 1999; 18:477-485.

6. Lang GA, Iwakuma T, Suh YA, Liu G, Rao VA, Parant JM, Valentin-Vega YA, Terzian T, Caldwell LC, Strong LC, ElNaggar AK, Lozano G. Gain of function of a p53 hot spot mutation in a mouse model of Li-Fraumeni syndrome. Cell. 2004; 119:861-872.

7. Wang XJ, Greenhalgh DA, Jiang A, He D, Zhong L, Brinkley BR, Roop DR. Analysis of centrosome abnormalities and angiogenesis in epidermal-targeted p53172H mutant and p53-knockout mice after chemical carcinogenesis: evidence for a gain of function. Mol Carcinog. 1998; 23:185-192.

8. Vikhanskaya F, Lee MK, Mazzoletti M, Broggini M, Sabapathy K. Cancer-derived p53 mutants suppress p53-target gene expression - potential mechanism for gain of function of mutant p53. Nucleic Acids Res. 2007; 35:2093-2104.

9. Scian MJ, Stagliano KE, Ellis MA, Hassan S, Bowman M, Miles MF, Deb SP, Deb S. Modulation of gene expression by tumor-derived p53 mutants. Cancer Res. 2004; 64:7447-7454.

10. Elston R, Inman GJ. Crosstalk between p53 and TGF-beta Signalling. J Signal Transduct. 2012; 2012:294097.

11. Adorno M, Cordenonsi M, Montagner M, Dupont S, Wong C, Hann B, Solari A, Bobisse S, Rondina MB, Guzzardo V, Parenti AR, Rosato A, Bicciato S, et al. A Mutant-p53/ Smad complex opposes p63 to empower TGFbeta-induced metastasis. Cell. 2009; 137:87-98.

12. Dupont S, Zacchigna L, Adorno M, Soligo S, Volpin D, Piccolo S, Cordenonsi M. Convergence of p53 and TGFbeta signaling networks. Cancer Lett. 2004; 213:129-138.

13. Ji L, Xu J, Liu J, Amjad A, Zhang K, Liu Q, Zhou L, Xiao J, Li X. Mutant p53 promotes tumor cell malignancy by both positive and negative regulation of the transforming growth factor beta (TGF-beta) pathway. J Biol Chem. 2015; 290:11729-11740.

14. Cordenonsi M, Dupont S, Maretto S, Insinga A, Imbriano C, Piccolo S. Links between tumor suppressors: p53 is required for TGF-beta gene responses by cooperating with Smads. Cell. 2003; 113:301-314.

15. Muller PA, Vousden KH. p53 mutations in cancer. Nat Cell Biol. 2013; 15:2-8.

16. Zhu J, Sammons MA, Donahue G, Dou Z, Vedadi M, Getlik M, Barsyte-Lovejoy D, Al-awar R, Katona BW, Shilatifard A, Huang J, Hua X, Arrowsmith CH, et al. Gainof-function $\mathrm{p} 53$ mutants co-opt chromatin pathways to drive cancer growth. Nature. 2015; 525:206-211.

17. Ali A, Wang Z, Fu J, Ji L, Liu J, Li L, Wang H, Chen J, Caulin C, Myers JN, Zhang P, Xiao J, Zhang B, et al. Differential regulation of the REGgamma-proteasome pathway by $553 /$ TGF-beta signalling and mutant p53 in cancer cells. Nat Commun. 2013; 4:2667.

18. Murphy M, Ahn J, Walker KK, Hoffman WH, Evans RM, Levine AJ, George DL. Transcriptional repression by wild-type p53 utilizes histone deacetylases, mediated by interaction with mSin3a. Genes Dev. 1999; 13:2490-2501.

19. Juan LJ, Shia WJ, Chen MH, Yang WM, Seto E, Lin YS, $\mathrm{Wu} \mathrm{CW}$. Histone deacetylases specifically down-regulate p53-dependent gene activation. J Biol Chem. 2000; 275:20436-20443.

20. Di Agostino S, Strano S, Emiliozzi V, Zerbini V, Mottolese M, Sacchi A, Blandino G, Piaggio G. Gain of function of mutant $\mathrm{p} 53$ : the mutant $\mathrm{p} 53 / \mathrm{NF}-\mathrm{Y}$ protein complex reveals an aberrant transcriptional mechanism of cell cycle regulation. Cancer Cell. 2006; 10:191-202.

21. Boudreau HE, Casterline BW, Burke DJ, Leto TL. Wildtype and mutant p53 differentially regulate NADPH oxidase 4 in TGF-beta-mediated migration of human lung and breast epithelial cells. Br J Cancer. 2014; 110:2569-2582.

22. Bai G, Hock TD, Logsdon N, Zhou Y, Thannickal VJ. A farupstream AP-1/Smad binding box regulates human NOX4 promoter activation by transforming growth factor-beta. Gene. 2014; 540:62-67.

23. Sanders YY, Liu H, Liu G, Thannickal VJ. Epigenetic mechanisms regulate NADPH oxidase-4 expression in cellular senescence. Free Radic Biol Med. 2015; 79:197-205.

24. Xu J, Lamouille S, Derynck R. TGF-beta-induced epithelial to mesenchymal transition. Cell Res. 2009; 19:156-172.

25. Sun XX, Dai MS, Lu H. 5-fluorouracil activation of $\mathrm{p} 53$ involves an MDM2-ribosomal protein interaction. J Biol Chem. 2007; 282:8052-8059.

26. Golubovskaya VM, Finch R, Kweh F, Massoll NA, Campbell-Thompson M, Wallace MR, Cance WG. p53 regulates FAK expression in human tumor cells. Mol Carcinog. 2008; 47:373-382.

27. Riley T, Sontag E, Chen P, Levine A. Transcriptional control of human p53-regulated genes. Nat Rev Mol Cell Biol. 2008; 9:402-412.

28. Kaustov L, Yi GS, Ayed A, Bochkareva E, Bochkarev A, Arrowsmith CH. p53 transcriptional activation domain: a molecular chameleon? Cell Cycle. 2006; 5:489-494.

29. Venot C, Maratrat M, Sierra V, Conseiller E, Debussche L. Definition of a 553 transactivation function-deficient mutant and characterization of two independent p53 transactivation subdomains. Oncogene. 1999; 18:2405-2410.

30. Murphy M, Hinman A, Levine AJ. Wild-type p53 negatively regulates the expression of a microtubule-associated protein. Genes Dev. 1996; 10:2971-2980.

31. Ropero S, Esteller M. The role of histone deacetylases (HDACs) in human cancer. Mol Oncol. 2007; 1:19-25.

32. McManus KJ, Hendzel MJ. Quantitative analysis of CBPand $\mathrm{P} 300$-induced histone acetylations in vivo using native chromatin. Mol Cell Biol. 2003; 23:7611-7627. 
33. Paladino D, Yue P, Furuya H, Acoba J, Rosser CJ, Turkson J. A novel nuclear Src and p300 signaling axis controls migratory and invasive behavior in pancreatic cancer. Oncotarget. 2016; 7:7253-7267. doi: 10.18632/oncotarget.6635.

34. Boudreau HE, Casterline BW, Rada B, Korzeniowska A, Leto TL. Nox4 involvement in TGF-beta and SMAD3driven induction of the epithelial-to-mesenchymal transition and migration of breast epithelial cells. Free Radic Biol Med. 2012; 53:1489-1499.

35. Boudreau HE, Emerson SU, Korzeniowska A, Jendrysik MA, Leto TL. Hepatitis C virus (HCV) proteins induce NADPH oxidase 4 expression in a transforming growth factor betadependent manner: a new contributor to HCV-induced oxidative stress. J Virol. 2009; 83:12934-12946.

36. Beckerman R, Prives C. Transcriptional regulation by $\mathrm{p} 53$. Cold Spring Harb Perspect Biol. 2010; 2:a000935.

37. Li Y, Seto E. HDACs and HDAC Inhibitors in Cancer Development and Therapy. Cold Spring Harb Perspect Med. $2016 ; 6$.
38. Gao J, Aksoy BA, Dogrusoz U, Dresdner G, Gross B, Sumer SO, Sun Y, Jacobsen A, Sinha R, Larsson E, Cerami E, Sander C, Schultz N. Integrative analysis of complex cancer genomics and clinical profiles using the cBioPortal. Sci Signal. 2013; 6:pl1.

39. Cerami E, Gao J, Dogrusoz U, Gross BE, Sumer SO, Aksoy BA, Jacobsen A, Byrne CJ, Heuer ML, Larsson E, Antipin Y, Reva B, Goldberg AP, et al. The cBio cancer genomics portal: an open platform for exploring multidimensional cancer genomics data. Cancer Discov. 2012; 2:401-404.

40. Team RDC. R: A Language and Environment for Statistical Computing. Vienna, Austria. 2016.

41. Morikawa M, Koinuma D, Miyazono K, Heldin $\mathrm{CH}$. Genome-wide mechanisms of Smad binding. Oncogene. 2013; 32:1609-1615. 